\title{
Logic-in-memory based on an atomically thin semiconductor
}

https://doi.org/10.1038/s41586-020-2861-0

Received: 15 January 2020

Accepted: 26 August 2020

Published online: 4 November 2020

Check for updates

\author{
Guilherme Migliato Marega ${ }^{1,2}$, Yanfei Zhao ${ }^{1,2}$, Ahmet Avsar ${ }^{1,2}$, Zhenyu Wang ${ }^{1,2}$, \\ Mukesh Tripathi ${ }^{1,2}$, Aleksandra Radenovic ${ }^{3}$ \& Andras Kis ${ }^{1,2 凶}$
}

The growing importance of applications based on machine learning is driving the need to develop dedicated, energy-efficient electronic hardware. Compared with von Neumann architectures, which have separate processing and storage units, braininspired in-memory computing uses the same basic device structure for logic operations and data storage ${ }^{1-3}$, thus promising to reduce the energy cost of data-centred computing substantially ${ }^{4}$. Although there is ample research focused on exploring new device architectures, the engineering of material platforms suitable for such device designs remains a challenge. Two-dimensional materials ${ }^{5,6}$ such as semiconducting molybdenum disulphide, $\mathrm{MoS}_{2}$, could be promising candidates for such platforms thanks to their exceptional electrical and mechanical properties ${ }^{7-9}$. Here we report our exploration of large-area $\mathrm{MoS}_{2}$ as an active channel material for developing logic-in-memory devices and circuits based on floating-gate field-effect transistors (FGFETs). The conductance of our FGFETs can be precisely and continuously tuned, allowing us to use them as building blocks for reconfigurable logic circuits in which logic operations can be directly performed using the memory elements. After demonstrating a programmable NOR gate, we show that this design can be simply extended to implement more complex programmable logic and a functionally complete set of operations. Our findings highlight the potential of atomically thin semiconductors for the development of next-generation low-power electronics.
Emerging data-intensive applications in fields including machine learning and the Internet of Things require highly energy-efficient hardware for operations such as autonomous driving ${ }^{10}$, speech recognition ${ }^{11}$ and disease diagnosis ${ }^{12}$. Because these specific applications require both high-performance and energy-efficient computation, the power ${ }^{13}$ and memory ${ }^{14}$ constraints imposed by von Neumann computers, with separate processing and storage units, limit the ability of standard processors to meet optimal requirements for these applications ${ }^{15}$. Next-generation architectures have therefore been an important subject of research ${ }^{16-19}$. Among them, in-memory computing, using the same basic device structure for logic operations and data storage ${ }^{1,2}$, is presenting itself as an ideal hardware architecture for tackling portable data-intensive ${ }^{20,21}$ and adaptive logic applications ${ }^{22}$. The success of this approach depends strongly on identifying an ideal material system capable of harnessing the full potential of this architecture.

Two-dimensional (2D) transition metal dichalcogenides (TMDs) have been considered as a candidate material system for realizing scaled semiconducting devices and circuits ${ }^{23}$ because of their atom-scale thickness, the absence of dangling bonds and enhanced electrostatic control ${ }^{7}$. Monolayer $\mathrm{MoS}_{2}$ in particular possesses a sizeable direct bandgap $^{24}$, enabling a strong modulation of the semiconducting channel with a high ON/OFF current ratio $\left(I_{\mathrm{ON}} / I_{\mathrm{OFF}} \approx 10^{8}\right)$, reduced standby current even at nanometre-scale gate lengths ${ }^{25}$ and a subthreshold slope approaching the theoretical limit ${ }^{7}$. This makes it an appealing choice for both next-generation logic circuits ${ }^{26,27}$ and memories in the form of FGFETs ${ }^{28-31}$, which are attractive devices for in-memory computing. In this context, 2D materials can enable aggressive scaling below $12 \mathrm{~nm}$ and at the same time also increase device reliability, thanks to the atom-scale thickness and reduced cell-to-cell interference between neighbouring thin-film floating gates in the FGFETs ${ }^{32}$.

2D materials therefore combine advantages for realizing both logic and memory. Their applications in neuromorphic computing are, however, rare and have been limited to single devices ${ }^{33-35}$. Moreover, overcoming device-to-device variation and large-area integration at the system level remain crucial to the realization of large-scale systems that could open the path to creating new, unexplored circuit functionalities.

Here we demonstrate the integration of $\mathrm{MoS}_{2}$ memories into a subsystem for in-memory computing, and demonstrate reprogrammable logic operations. The basic building block of our circuits are FGFETs with a monolayer $\mathrm{MoS}_{2}$ channel, allowing us to build simple logic-in-memory arrays ${ }^{1}$. Our $\mathrm{MoS}_{2}$ is grown using a large-grain, large-area metal-organic chemical vapour deposition (MOCVD) process ${ }^{36,37}$. Figure $1 \mathrm{a}, \mathrm{b}$ shows the floating-gate memory structure used throughout this work and its side-view schematic. Our device has a local $\mathrm{Cr} / \mathrm{Pd}(2 \mathrm{~nm} / 80 \mathrm{~nm})$ bottom gate and a thin-film Pt floating gate ( $5 \mathrm{~nm}$ thickness), which results in a continuous and smooth surface. The reduced roughness 


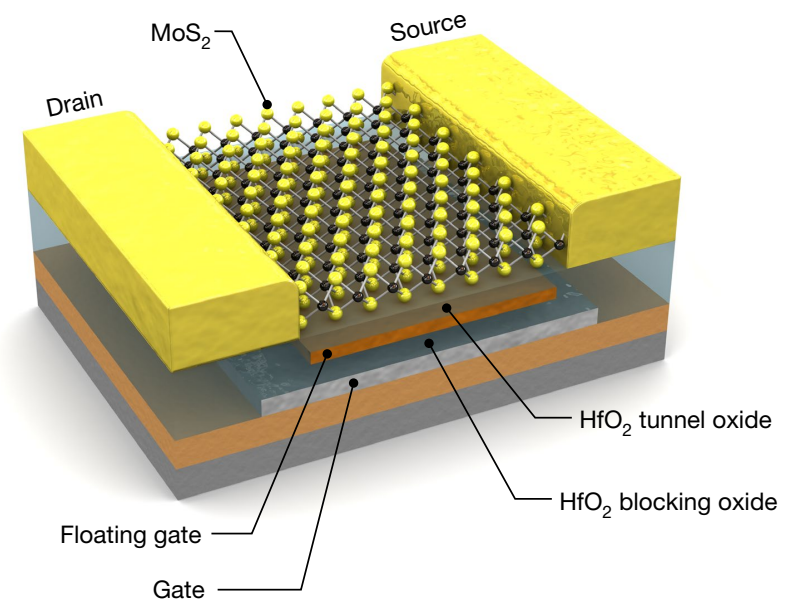

b
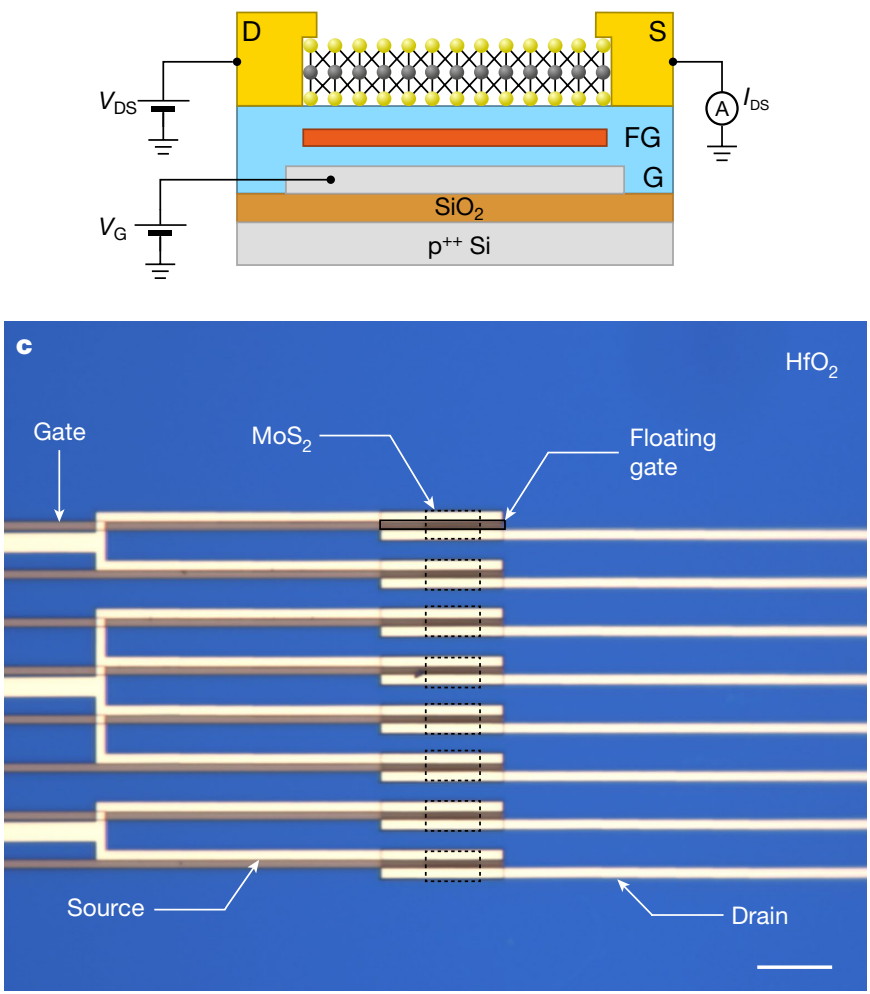

Fig. 1 Structure of in-memory device. a, Three-dimensional view of a floating-gate memory device based on MOCVD-grown monolayer $\mathrm{MoS}_{2}$ with source and drain contacts. The floating gate is separated from the $\mathrm{MoS}_{2}$ channel by a 7-nm-thick $\mathrm{HfO}_{2}$ tunnel oxide layer and from the bottom control gate by a 30-nm-thick $\mathrm{HfO}_{2}$ blocking oxide layer. $\mathbf{b}$, Schematic of the device. D, drain; S, source; FG, floating gate; $G$, gate.c, Optical image of the fabricated floating-gate memory array, comprising eight memory cells (boxed). Scale bar, $10 \mu \mathrm{m}$.

of the metal surface decreases the dielectric disorder in the interface between the top tunnel oxide and the 2D channel, improving performance and reliability ${ }^{38}$. Both blocking and tunnel oxides $(30 \mathrm{~nm}$ and $7 \mathrm{~nm}$ thick, respectively) consist of high- $\kappa$ dielectric $\mathrm{HfO}_{2}$ deposited by atomic-layer deposition (ALD) to achieve effective modulation of the electric field within the semiconducting channel. Finally, the contacts to the drain and source are composed of a Ti/Au $(2 \mathrm{~nm} / 100 \mathrm{~nm})$ stack to obtain ohmic-like contacts with high charge carrier injection efficiency. Figure 1c shows an optical micrograph of a fabricated memory array.
We note that all the device components are fabricated in an approach that is scalable, that is, no exfoliated materials were used.

\section{Floating-gate memory}

The FGFET memory behaviour manifests itself in a shift of the transistor threshold voltage controlled by the amount of charge stored in the charge trap layer (see Supplementary Note 1 for details). To read the memory state of the device, a constant voltage is applied to the gate $\left(V_{\mathrm{G}, \mathrm{READ}}\right)$ while the drain-source conductance is measured. We perform the basic characterization of our devices by sweeping the gate voltage in the range $\pm 12.5 \mathrm{~V}$ under a constant 50 - $\mathrm{mV}$ drain-source voltage $\left(V_{\mathrm{DS}}\right)$, Fig. 2a. The total shift of the memory threshold voltage $\left(V_{\mathrm{TH}}\right)$ gives an estimated memory window of $10.6 \mathrm{~V}$, taken for a 1-nA constant current. The linear behaviour of the drain-source current $\left(I_{\mathrm{DS}}\right)$ versus $V_{\mathrm{DS}}$ traces (Fig. 2b) indicates ohmic-like contacts. The same multilevel behaviour is illustrated in Fig. 2c, in which we show the ability to set the channel conductance with the programming voltage $\left(V_{\mathrm{PROG}}\right)$. Before applying the observed multilevel behaviour of our memory to in-memory computing, we check retention times to verify that the programmed conductance values are stable over time. In Fig. 2c, we show the evolution of the ON and OFF states of our memory as well as multiple intermediate states stable in a 1-h time frame, Fig. 2c. We project a retention time of about 10 years for two-state operation (see Extended Data Fig.1). Other critical memory characterization concerning device variability and memory behaviour under different constraints is provided in Extended Data Fig. 2 with band alignments shown in Extended Data Fig. 3.

In addition to programming the memory using the programming voltage $\left(V_{\mathrm{PROG}}\right)$, we can also fine-tune the conductance states to the desired level by applying short potentiative $\left(V_{\mathrm{G} \text {,PEAK }}=-5 \mathrm{~V}\right)$ and depressive $\left(V_{\mathrm{G}, \mathrm{PEAK}}=5 \mathrm{~V}\right)$ pulses with a 10 -ms pulse width and 1-s rest time, allowing a finer control over the device conductance. Figure $2 \mathrm{~d}$ shows the linear evolution of the conductance values for potentiation (which can be used to rapidly set the desired conductance value) and for depressive stages (to reset the memory state). Results of the endurance test (Supplementary Note 2), shown in Extended Data Fig. 4, demonstrate that our memories can sustain more than 10,000 programming pulses with no performance loss.

\section{Programmable inverter}

As shown in Fig. 3a, using FGFETs as the basic building blocks instead of normal FETs brings us the capability of programming the threshold voltage, giving an additional degree of freedom for applications in both digital and analogue circuits. The gate terminal can then be used for both setting the state of the memory using a programming voltage $V_{\mathrm{PROG}}$ and as a terminal for applying the input voltage $\left(V_{\mathrm{IN}}\right)$ during logic operations.

We take advantage of the fine control over the conductance states of the 2D material, and tune the memory cell's threshold voltage by adding or removing charge carriers from the floating gate. This enables different electron transport regimes to be accessed when the memory is operated in the inverter circuit. We limit the gate voltage during regular operation $\left(V_{\mathrm{G}}=V_{\mathrm{IN}}\right)$ to a range of $0-1 \mathrm{~V}$, corresponding to logic ' 0 ' and ' 1 '. With this, we can avoid programming currents and preserve the pre-programmed memory state $(Q)$. The output voltage $V_{\text {OUT }}$ and the corresponding logic state are defined by both the logic input and the memory logic state $X^{(Q)}$. The relationship between them is shown in the tables in Fig. 3a. As presented in Fig. 3b-d, we can differentiate between three distinct and discrete states of the memory device according to how efficiently the gate electrode is screened by the charges present in the floating gate. For states $Q=1$ and 3 , the charges present in the floating gate strongly dope the FGFET channel which remains in the OFF $(Q=1)$ or ON $(Q=3)$ states for all values of $V_{\mathrm{IN}}$ in the $0-1 \mathrm{~V}$ range. The output then becomes independent of the input and the memory logic 


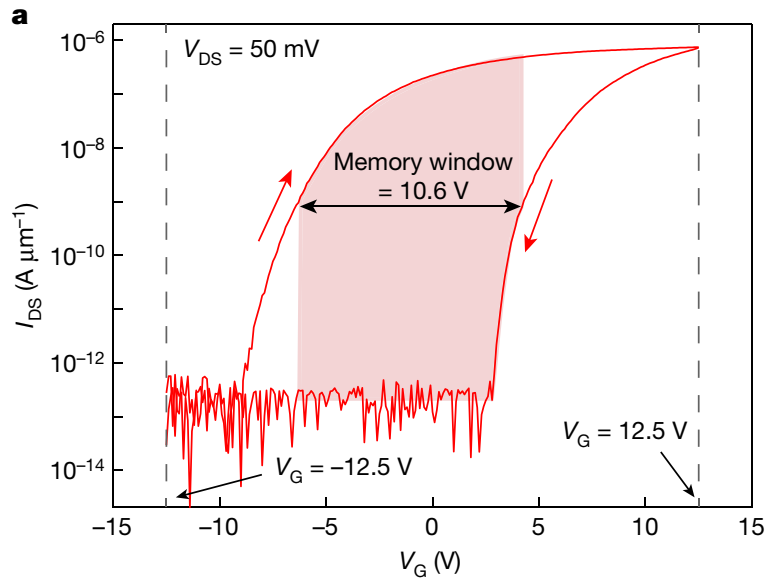

c

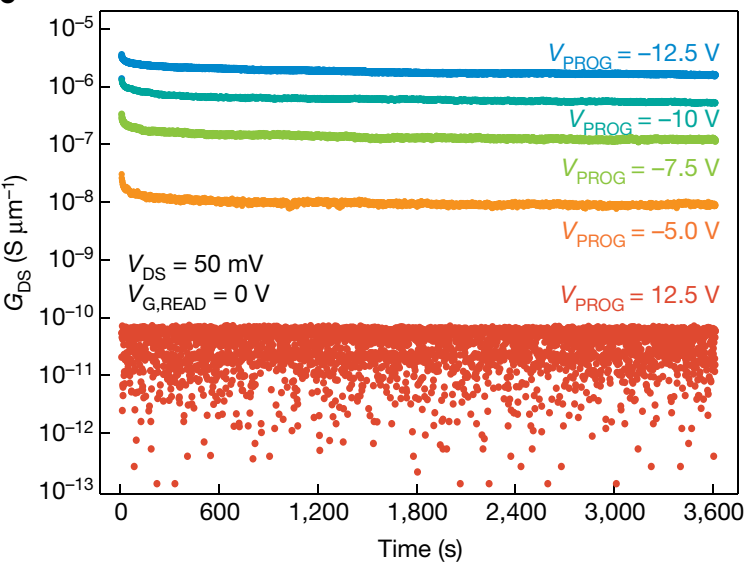

Fig. 2 Characterization of non-volatile memories. a, Transfer characteristic ( $I_{\mathrm{DS}}$ versus $V_{\mathrm{G}}$ ) of the FGFET acquired for two different gate voltage sweep directions (red arrows). The variation of the threshold voltage $V_{\mathrm{TH}}$, the memory window, is estimated to be $10.6 \mathrm{~V}$. b, Output characteristics ( $I_{\mathrm{DS}}$ versus $\left.V_{\mathrm{DS}}\right)$ of the FGFET in the ON state, after having been programmed using different values of the programming voltage, $V_{\mathrm{PROG}}$, for $V_{\mathrm{DS}}=50 \mathrm{mV}, V_{\mathrm{G}, \mathrm{READ}}=0 \mathrm{~V}$. c, Time dependence of the device conductance for different levels of programming voltage, $V_{\text {PROG }}$. These retention measurements show multilevel states of the normalized conductance $G_{\mathrm{DS}}$ versus time as a function of the programming

states are 0 (for $Q=1$ ) or 1 (for $Q=3$ ). For $Q=2$, the amount of charge stored on the floating gate is insufficient for inhibiting the channel modulation and $V_{\mathrm{TH}}$ is tuned to be in the $0-1 \mathrm{~V}$ range. Here, the memory cell functions as a normal FET with a programmable threshold voltage. In this case, the memory state reflects the input logic state $\left(X^{(2)}=\mathrm{IN}\right)$ and the circuit operates as an inverter.

The programmable shift in the threshold $V_{\mathrm{TH}}$ allows us to fine-tune the transfer curve of the inverter circuit, shown in Fig. 3e for programming voltages in the $7.5-9 \mathrm{~V}$ range. The gain and noise margin of the circuit can also be configured for either a more precise (lower noise margin) or a more robust circuit (higher noise margin). Figure $3 \mathrm{f}$ show the evolution of both high $\left(\mathrm{NM}_{\mathrm{H}}\right)$ and low $\left(\mathrm{NM}_{\mathrm{L}}\right)$ noise margin as a function of the programming voltage $\left(V_{\mathrm{PROG}}\right.$; see Supplementary Note 3 , Supplementary Table 1 and Extended Data Fig. 5 for details). Time traces displayed in Fig. $3 g$ show that the different configurations of the circuit are stable and reproducible.

\section{Logic-in-memory}

This multitude of memory states (always ON/always OFF and a normal FET) opens a way to configure memory arrays as a large set of distinct logic circuits. When multiple FGFETs are assembled into a logic gate,

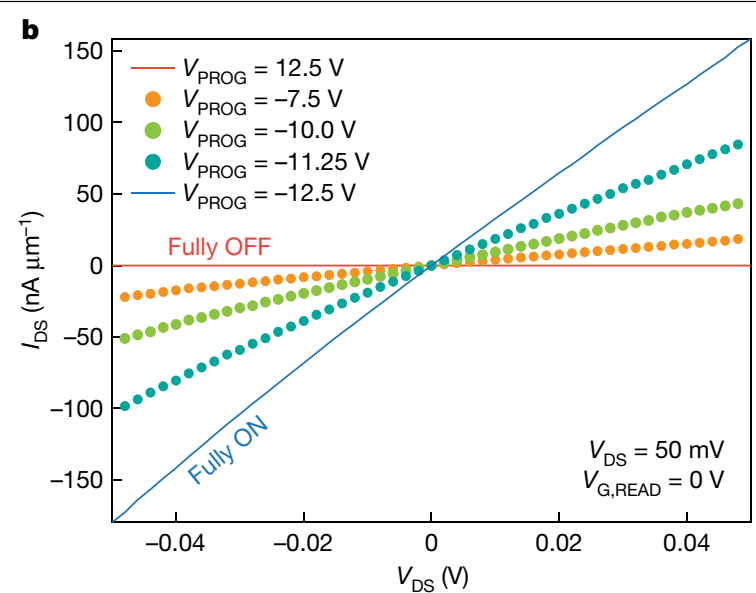

d

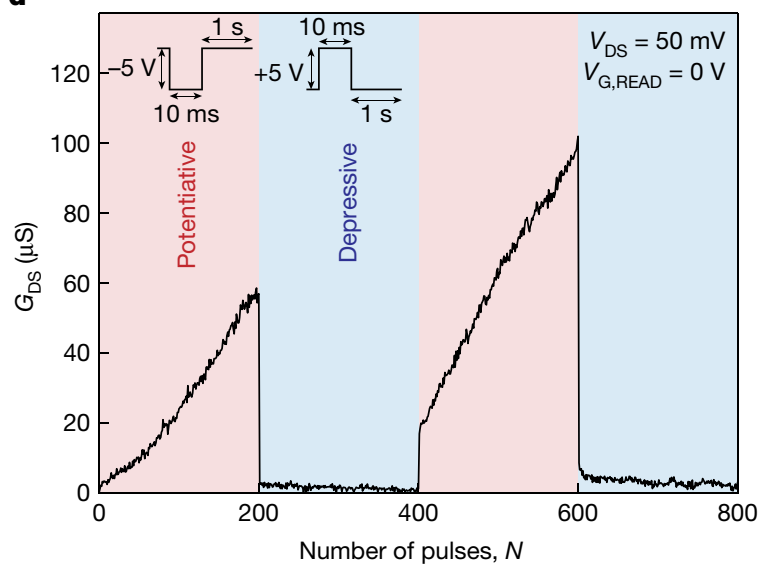

voltage, $V_{\mathrm{PROG}}($ ranging from $-12.5 \mathrm{~V}$ to $12.5 \mathrm{~V}$ ). d, Demonstration of fine control over the conductance state of the memory using voltage pulses applied to the gate. Shown is the evolution of the memory device conductance $G_{\mathrm{DS}}$ as a function of the number of pulses. Potentiative pulses applied to the gate ( $-5 \mathrm{~V}$ amplitude, $10 \mathrm{~ms}$ duration and $1 \mathrm{~s}$ rest time) can be used to increase and fine-tune the conductance. Depressive pulses ( $+5 \mathrm{~V}$ amplitude, $10 \mathrm{~ms}$ duration and $1 \mathrm{~s}$ rest time) can reset the device. The conductance measurement is performed at the end of the rest time period. Before the first pulse, the memory was initialized with a reset voltage $V_{\mathrm{PROG}}=12.5 \mathrm{~V}$.

the number of possible functions grows exponentially with the number of devices (see Supplementary Note 4). To demonstrate this principle, we show that simple logic gates (two-input NOR and three-input NOR) can be implemented using two or three devices, and can have their functionality expanded up to nine different Boolean functions (see Supplementary Note 4, Supplementary Tables 2,3 and Extended Data Figs. 6, 7). These new logic operations emerge as subsets of the main function. For instance, a three-input NOR gate contains a two-input NOR and NOT as subset operations. Hence, the circuit area per functionality is greatly decreased as the circuit size grows.

We take advantage of this large number of available logic functions to propose in Fig. 4a a two-input logic-in-memory unit cell capable of acting as a universal logic gate, performing any logic operation from a complete set of two-input logic operations (Extended Data Fig. 8 and Supplementary Note 5). By combining two cells, we can perform more complex operations, such as the addition of two numbers using a half-adder, shown in Fig. 4b (see Supplementary Tables 4, 5 for a breakdown of available functions). This is made possible by adding polarity control in the input and output of the cell. With this new degree of freedom, one unit operates as an XOR logic gate producing as a result the binary SUM $(S)$ and the second unit producing the logic NAND which after inversion by the output interface generates 


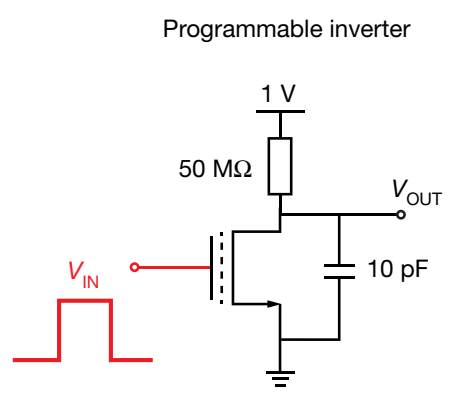

\begin{tabular}{|c|c|c|c|c|c|c|}
\hline$Q$ & \multicolumn{2}{|c|}{ IN } & \multicolumn{2}{|c|}{$X^{(Q)}$} & $X^{(Q)}$ & OUT \\
\hline 1 & 0 & 1 & 0 & 0 & 0 & 1 \\
\hline 2 & 0 & 1 & 0 & 1 & 1 & 0 \\
\hline 3 & 0 & 1 & 1 & 1 & & \\
\hline
\end{tabular}

Logic input and memory states Memory states and logic output

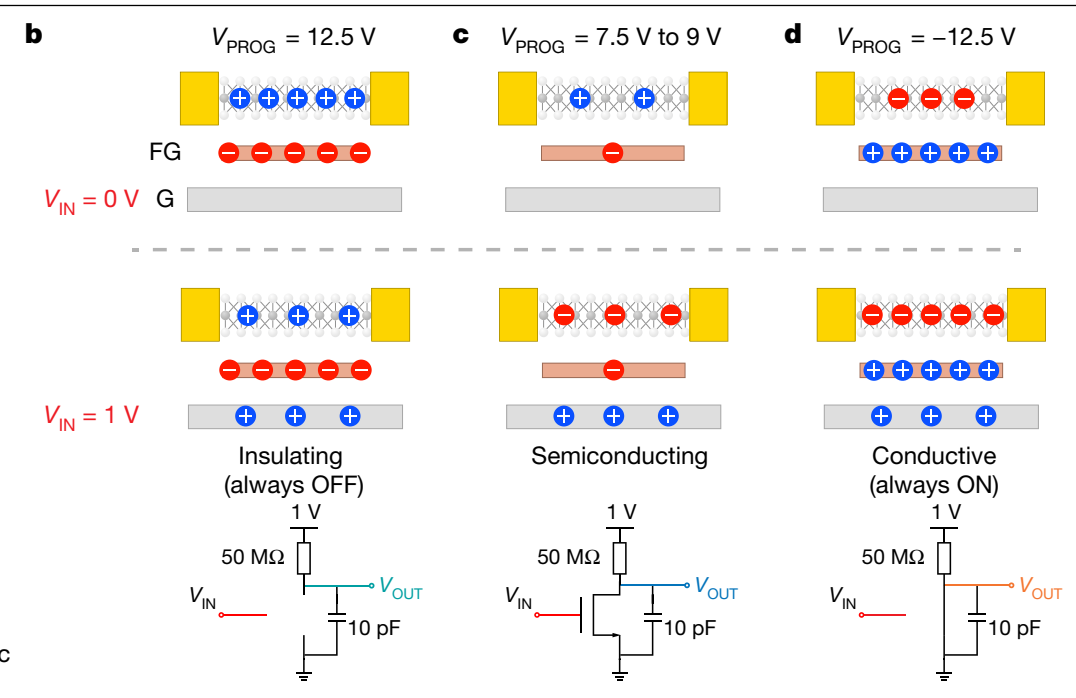

$Q=1$ : memory state 0

$Q=2$ : memory state 0 or 1

$Q=3$ : memory state 1 e

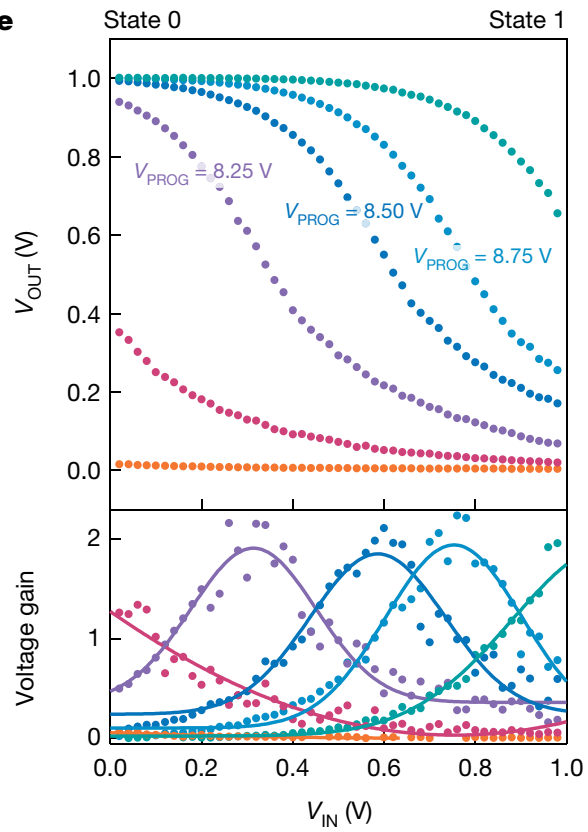

$\mathbf{f}$

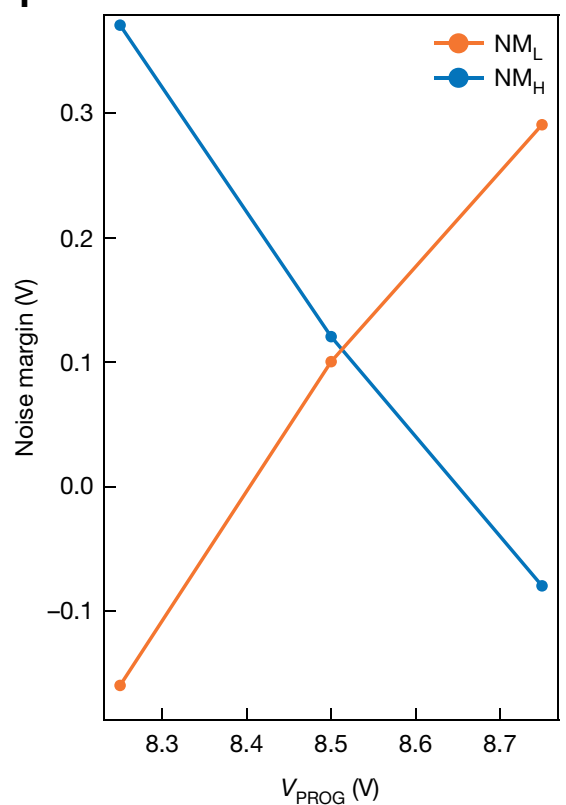

$\mathbf{g}$

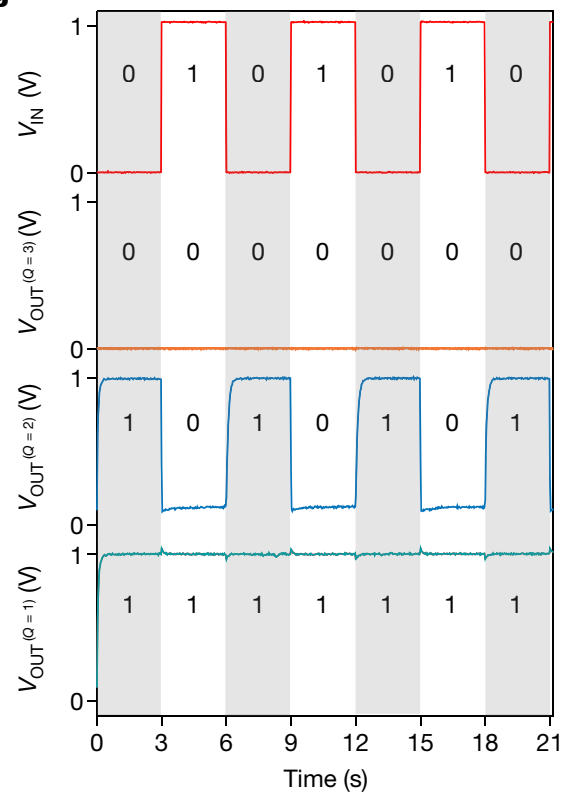

Fig. 3 | Programmable inverter based on a $\mathbf{M o S}_{2}$ memory cell. a, Schematic of the programmable inverter (top), different states of the device (bottom left) and the inverter truth table (bottom right). b-d, Illustration of the discrete memory states used for programmable behaviour and (bottom) the corresponding circuit diagrams. Device elements shown are the channel, source and drain electrodes (yellow blocks), floating gate (orange bar) and control gate (grey bar), with stored and induced extra positive (blue) and negative (red) charges. $\mathbf{b}$, Insulating state of the $\mathrm{MoS}_{2}$ channel $(Q=1$, memory state 0 ).c, Semiconducting state with a continuously tunable conductance of the $\mathrm{MoS}_{2}$ channel $(Q=2$, memory states 0 or 1$)$. d, Conductive state of the channel $(Q=3$, memory state 1$)$. e, Programmable output $\left(V_{\text {OUT }}\right)$ curves of the inverter (top) and the inverter voltage gain (bottom) both as a function of the input voltage $\left(V_{\mathrm{IN}}\right)$, for different programming conditions.f, Evolution of the inverter noise margin $\left(\mathrm{NM}_{\mathrm{L}}\right.$ and $\left.\mathrm{NM}_{\mathrm{H}}\right)$ as a function of the programming voltage.g, Time traces showing stability of the output voltage for the three different configurations of the programmable inverter. Red, input voltage $\left(V_{\text {IN }}\right)$; orange, constant memory state $1(Q=3)$; blue, inverter operation; green, constant memory state $0(Q=1)$. the CARRY $(C)$ value. Because the half-adder is a basic building block in modern processors, this shows that logic-in-memory based on 2D materials could be extended to complex computational accelerators. In contrast to current logic-in-memory circuits ${ }^{39}$ (see also Supplementary Note 6 and Supplementary Table 6), our approach allows cascading different cells without the need for complex current-voltage conversion circuits. This eliminates the extra power consumption and enables the creation of more complex circuits similar to modern CMOS digital processors. Logic-in-memory units can be connected in parallel to execute more complex operations, and the signal can be transferred to the next set of units, creating a structure like a field-programmable gate array.
To archive higher parallelism and more complex operation, the number of logic inputs can be further increased. As shown in Fig. 4a, the concept of a three-input cell increases the functionality that can be implemented compared to a two-input structure. As a proof of concept, we show in Fig. 4c a three-input cell operating in one of its possible states, three-input NAND, with the corresponding transfer curves for individual memory elements shown in Extended Data Fig. 9.

We have demonstrated here reprogrammable logic devices for in-memory processing architectures based on monolayer $\mathrm{MoS}_{2}$. By employing an innovative way of realizing a universal logic gate based on logic-in-memory, we have produced a programmable logic circuit that operates directly in memory and does not require additional terminals 
a

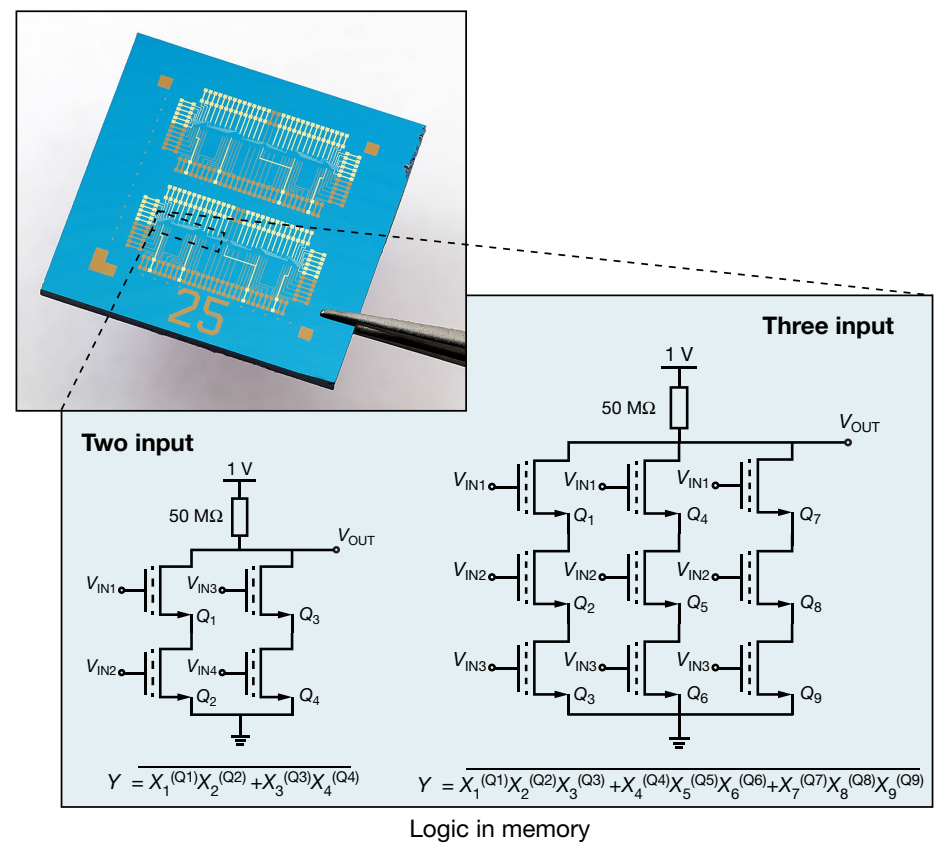

c

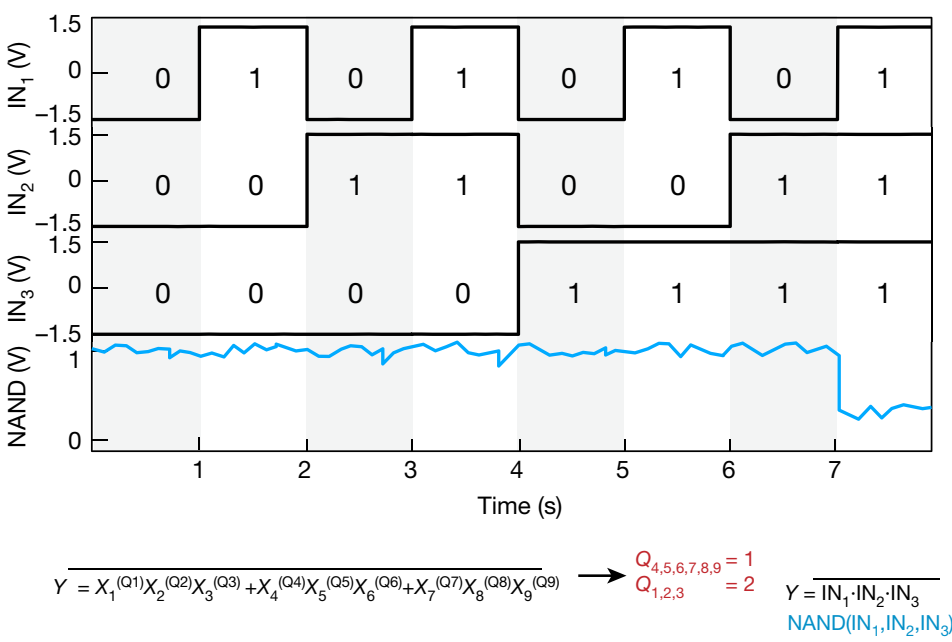

b

Half-adder

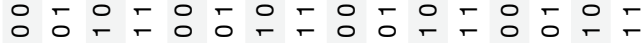

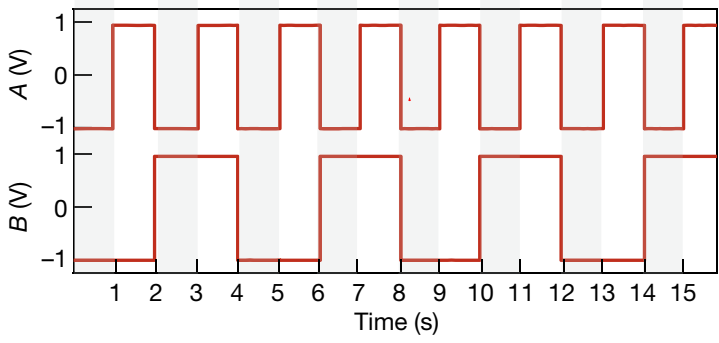

Input logic
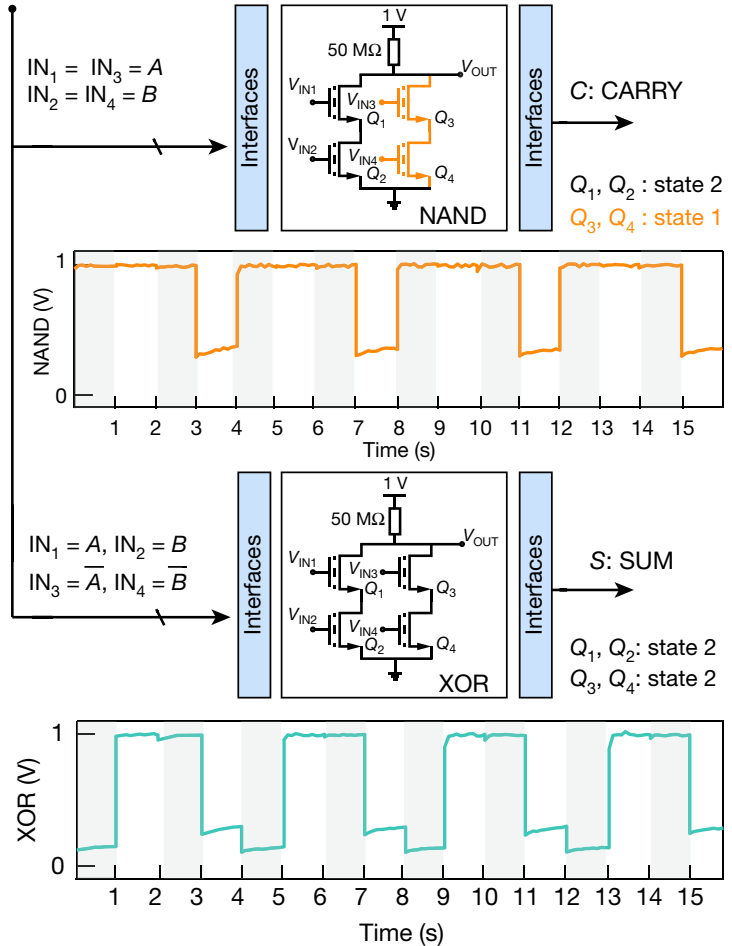

Fig. 4 | Logic-in-memory. a, Top left, photograph of a fabricated $12 \mathrm{~mm} \times 12 \mathrm{~mm}$ die with logic-in-memory cell arrays. The boxed area contains two-input and three-input logic-in-memory cells, and their schematics are shown below with $Y$ the output logic function. b, System level operation of 2 two-input cells ('Input logic', bottom) to form a half-adder (top). XOR is programmed as $Q_{1-4}=2$ with inputs to memories $Q_{3}$ and $Q_{4}$ inverted. NAND is programmed as $Q_{1,2}=2, Q_{3,4}=1$, and output is inverted to form the AND logic operation. $c$, Time traces showing stability of the output voltage for the NAND operation of the three-input unit cell. For this configuration, memories are programmed as follows: $Q_{1-3}=2, Q_{4-9}=1$. The transfer curves of each state can be seen in Extended Data Fig 9. for programming ${ }^{40,41}$. This direct integration of memory and logic can increase processing speed, opening the way to the realization of energy-efficient circuits based on 2D materials for machine learning, the Internet of Things and non-volatile computing.

\section{Online content}

Any methods, additional references, Nature Research reporting summaries, source data, extended data, supplementary information, acknowledgements, peer review information; details of author contributions and competing interests; and statements of data and code availability are available at https://doi.org/10.1038/s41586-020-2861-0.

1. Kautz, W. H. Cellular logic-in-memory arrays. IEEE Trans. Comput. C-18, 719-727 (1969).

2. Stone, H. S. A logic-in-memory computer. IEEE Trans. Comput. C-19, 73-78 (1970)

3. Le Gallo, M. et al. Mixed-precision in-memory computing. Nat. Electron. 1, 246-253 (2018).
4. Horowitz M. Computing's energy problem (and what we can do about it). In 2014 IEEE Intl Solid-State Circuits Conf. Digest of Technical Papers (ISSCC) 10-14 (IEEE, 2014).

5. Wang, Q. H., Kalantar-Zadeh, K., Kis, A., Coleman, J. N. \& Strano, M. S. Electronics and optoelectronics of two-dimensional transition metal dichalcogenides. Nat. Nanotechnol. 7, 699-712 (2012).

6. Manzeli, S., Ovchinnikov, D., Pasquier, D., Yazyev, O. V. \& Kis, A. 2D transition metal dichalcogenides. Nat. Rev. Mater. 2, 17033 (2017)

7. Radisavljevic, B., Radenovic, A., Brivio, J., Giacometti, V. \& Kis, A. Single-layer $\mathrm{MoS}_{2}$ transistors. Nat. Nanotechnol. 6, 147-150 (2011).

8. Bertolazzi, S., Brivio, J. \& Kis, A. Stretching and breaking of ultrathin $\mathrm{MoS}_{2}$. ACS Nano 5 , 9703-9709 (2011).

9. Iannaccone, G., Bonaccorso, F., Colombo, L. \& Fiori, G. Quantum engineering of transistors based on 2D materials heterostructures. Nat. Nanotechnol. 13, 183-191 (2018); correction 15,520 (2018)

10. Wu, B., Wan, A., landola, F., Jin, P. H. \& Keutzer, K. SqueezeDet: unified, small, low power fully convolutional neural networks for real-time object detection for autonomous driving. In 2017 IEEE Conf. Computer Vision and Pattern Recognition Workshops (CVPRW) 446-454 (IEEE, 2017).

11. Graves, A., Mohamed, A. \& Hinton, G. Speech recognition with deep recurrent neural networks. In 2013 IEEE Intl Conf. Acoustics, Speech and Signal Processing 6645-6649 (IEEE, 2013). 
12. Kononenko, I. Machine learning for medical diagnosis: history, state of the art and perspective. Artif. Intell. Med. 23, 89-109 (2001).

13. Naffziger, S. High-performance processors in a power-limited world. In 2006 Symp. VLS Circuits, 2006. Digest of Technical Papers 93-97 (IEEE, 2006).

14. McKee, S. A. Reflections on the memory wall. In Proc. First Conf. Computing Frontiers CF'04 162 (ACM Press, 2004).

15. $\mathrm{Xu}, \mathrm{X}$. et al. Scaling for edge inference of deep neural networks. Nat. Electron. 1, 216-222 (2018).

16. Arute, F. et al. Quantum supremacy using a programmable superconducting processor. Nature 574, 505-510 (2019).

17. Kwon, J. et al. Three-dimensional monolithic integration in flexible printed organic transistors. Nat. Commun. 10, 54 (2019).

18. Shulaker, M. M. et al. Three-dimensional integration of nanotechnologies for computing and data storage on a single chip. Nature 547, 74-78 (2017).

19. Yu, S. Neuro-inspired computing with emerging nonvolatile memories. Proc. IEEE 106, 260-285 (2018).

20. Chen, W.-H. et al. CMOS-integrated memristive non-volatile computing-in-memory for Al edge processors. Nat. Electron. 2, 420-428 (2019).

21. Li, C. et al. Analogue signal and image processing with large memristor crossbars. Nat. Electron. 1, 52-59 (2018).

22. Fuller, E. J. et al. Parallel programming of an ionic floating-gate memory array for scalable neuromorphic computing. Science 364, 570-574 (2019).

23. Fiori, G. et al. Electronics based on two-dimensional materials. Nat. Nanotechnol. 9, 768779 (2014); erratum 9, 1063 (2014).

24. Splendiani, A. et al. Emerging photoluminescence in monolayer $\mathrm{MoS}_{2}$. Nano Lett. 10, 1271-1275 (2010).

25. Desai, S. B. et al. $\mathrm{MoS}_{2}$ transistors with 1-nanometer gate lengths. Science 354, 99-102 (2016).

26. Radisavljevic, B., Whitwick, M. B. \& Kis, A. Integrated circuits and logic operations based on single-layer $\mathrm{MoS}_{2}$. ACS Nano 5, 9934-9938 (2011).

27. Wachter, S., Polyushkin, D. K., Bethge, O. \& Mueller, T. A microprocessor based on a two-dimensional semiconductor. Nat. Commun. 8, 14948 (2017).

28. Bertolazzi, S., Krasnozhon, D. \& Kis, A. Nonvolatile memory cells based on $\mathrm{MoS}_{2} /$ graphene heterostructures. ACS Nano 7, 3246-3252 (2013).
29. Li, D. et al. Nonvolatile floating-gate memories based on stacked black phosphorus-boron nitride-MoS ${ }_{2}$ heterostructures. Adv. Funct. Mater. 25, 7360-7365 (2015).

30. Tan, C., Liu, Z., Huang, W. \& Zhang, H. Non-volatile resistive memory devices based on solution-processed ultrathin two-dimensional nanomaterials. Chem. Soc. Rev. 44, 26152628 (2015).

31. Wang, J. et al. Floating gate memory-based monolayer $\mathrm{MoS}_{2}$ transistor with metal nanocrystals embedded in the gate dielectrics. Small 11, 208-213 (2015).

32. Cao, W., Kang, J., Bertolazzi, S., Kis, A. \& Banerjee, K. Can 2D-nanocrystals extend the lifetime of floating-gate transistor based nonvolatile memory? IEEE Trans. Electron Dev. 61, 3456-3464 (2014).

33. Sebastian, A., Pannone, A., Subbulakshmi Radhakrishnan, S. \& Das, S. Gaussian synapses for probabilistic neural networks. Nat. Commun. 10, 4199 (2019).

34. Sivan, M. et al. All WSe $\mathrm{C}_{2} 1 \mathrm{~T} 1 \mathrm{R}$ resistive RAM cell for future monolithic $3 \mathrm{D}$ embedded memory integration. Nat. Commun. 10, 5201 (2019).

35. Sun, L. et al. Self-selective van der Waals heterostructures for large scale memory array. Nat. Commun. 10, 3161 (2019).

36. Kim, H., Ovchinnikov, D., Deiana, D., Unuchek, D. \& Kis, A. Suppressing nucleation in metal-organic chemical vapor deposition of $\mathrm{MoS}_{2}$ monolayers by alkali metal halides. Nano Lett. 17, 5056-5063 (2017).

37. Cun, H. et al. Wafer-scale MOCVD growth of monolayer $\mathrm{MoS}_{2}$ on sapphire and $\mathrm{SiO}_{2}$. Nano Res. 12, 2646-2652 (2019).

38. Raja, A. et al. Dielectric disorder in two-dimensional materials. Nat. Nanotechnol. 14, 832-837 (2019).

39. Papandroulidakis, G., Vourkas, I., Vasileiadis, N. \& Sirakoulis, G. Ch. Boolean logic operations and computing circuits based on memristors. IEEE Trans. Circuits Syst. I/ 61, 972-976 (2014).

40. Resta, G. V. et al. Polarity control in $\mathrm{WSe}_{2}$ double-gate transistors. Sci. Rep. 6, 29448 (2016).

41. Resta, G. V. et al. Doping-free complementary logic gates enabled by two-dimensional polarity-controllable transistors. ACS Nano 12, 7039-7047 (2018).

Publisher's note Springer Nature remains neutral with regard to jurisdictional claims in published maps and institutional affiliations.

(c) The Author(s), under exclusive licence to Springer Nature Limited 2020 


\section{Methods}

\section{Material synthesis}

Single-crystal monolayer $\mathrm{MoS}_{2}$ was grown in a home-built system using the metal-organic chemical vapour deposition (MOCVD) method. C-plane sapphire was used as the growth substrate and annealed at $1,000^{\circ} \mathrm{C}$ to achieve an atomically smooth surface, necessary for epitaxial growth ${ }^{42}$. Before growth, the substrate is spin-coated with $\mathrm{NaCl}$ solution to suppress nucleation and promote growth ${ }^{36,37}$. The two precursors (molybdenum hexacarbonyl, $\mathrm{Mo}(\mathrm{CO})_{6}$, and hydrogen sulfide, $\mathrm{H}_{2} \mathrm{~S}$ ), with a flow rate ratio of $1: 6,028$, were carried by Ar gas to the MOCVD chamber and underwent reaction at $820^{\circ} \mathrm{C}$ for $30 \mathrm{~min}$. $\mathrm{Mo}(\mathrm{CO})_{6}$ was kept at $15^{\circ} \mathrm{C}$ in a water bath and the valve was closed immediately after the growth process, while $\mathrm{H}_{2} \mathrm{~S}$ continued flowing during the cooling process. Throughout the whole growth process, the furnace was kept at 850 mbar pressure. Raman spectroscopy confirmed the monolayer nature of the grown material (Extended Data Fig. 10), and transmission electron microscopy (TEM) imaging also indicated the high quality of the material (Extended Data Fig. 11).

Continuous, 2-inch wafer-scale monolayer $\mathrm{MoS}_{2}$ film for complex circuits shown in Fig. 4 was synthesized using MOCVD ${ }^{37}$. Similarly to the synthesis of single crystals, we anneal the sapphire wafers in air and coat them with a $0.2 \mathrm{~mol} \mathrm{I}^{-1}$ sodium chloride $(\mathrm{NaCl})$ solution in deionized water. The growth process lasts for $30 \mathrm{~min}$ in a quartz tube at atmospheric pressure and a temperature of $870{ }^{\circ} \mathrm{C}$. We use $\mathrm{Mo}(\mathrm{CO})_{6}$ and diethyl sulphide $\left(\left(\mathrm{C}_{2} \mathrm{H}_{5}\right)_{2} \mathrm{~S}\right)$ as precursors. An argon/hydrogen mixture is used as a carrier gas, delivered with flow rates of $210 \mathrm{~cm}^{3} \mathrm{STP} \mathrm{min}^{-1} /$

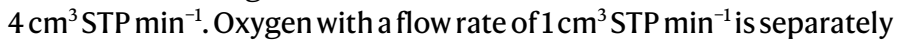
introduced into the growth chamber, for the purpose of balancing the growth rate with the $\mathrm{O}_{2}$ etching effect.

\section{Sample transfer and TEM imaging}

The sample was spin-coated with PMMA (poly(methyl methacrylate)) A2 at a speed of $4,000 \mathrm{rpm}$ for $1 \mathrm{~min}$ and put on a hot plate at $85^{\circ} \mathrm{C}$ for 10 min for drying. Afterwards, the PMMA film was detached with water tension and floated on the water's surface together with the $\mathrm{MoS}_{2}$ sample. Subsequently, the PMMA film was fished out using a TEM grid and heated for $15 \mathrm{~min}$ on a hot plate at $85^{\circ} \mathrm{C}$ to improve the adhesion. To remove the PMMA film, the sample was immersed in acetone overnight and annealed at $250^{\circ} \mathrm{C}$ in high vacuum for $6 \mathrm{~h}$.

Atomic-resolution annular dark field scanning TEM (ADF-STEM) images were acquired with an aberration-corrected (with double Cs corrector) FEI Titan Themis TEM operating at 60-300 kV, equipped with a Schottky X-FEG electron source and a monochromator. Imaging was performed at a low acceleration voltage $(80 \mathrm{kV})$. The electron probe semi-convergence angle was set to $21.2 \mathrm{mrad}$ and the typical beam current was $18 \mathrm{pA}$. Images were acquired with a Gatan high-angle annular angular dark-field (HAADF) detector using a $185 \mathrm{~mm}$ camera length which corresponds to a 49.5-198 mrad collection angle. To reduce sample drift distortion, a short dwell time $(8 \mu \mathrm{s})$ with $512 \times 512$ pixels was used to capture the frames. Cross-section lamellae were prepared using a focused ion beam (Zeiss NVision 40). TEM cross-sectional imaging was performed with a FEI Talos F200S G2, using $80 \mathrm{kV}$ acceleration voltage. Multislice STEM image simulations were performed using quantitative scanning transmission electron microscopy (QSTEM). The simulation parameters were chosen to be similar to the experimental conditions and higher-order aberrations were reduced to zero.

\section{Transfer procedure}

The MOCVD-grown material is first spin-coated with PMMA A2 at $1,500 \mathrm{rpm}$ for $60 \mathrm{~s}$. It is then dried in vacuum for $12 \mathrm{~h}$. After that, with the support of PDMS (polydimethylsiloxane) and Gel-pak elastomer film, the $\mathrm{MoS}_{2}$ sample is detached from the sapphire in deionized water and transferred onto the patterned substrate. Finally, the sample is immersed in acetone, and subsequently annealed at $250^{\circ} \mathrm{C}$ in high vacuum to remove the polymer resist.

\section{Memory fabrication}

A 270-nm-thick $\mathrm{SiO}_{2}$ layer is thermally grown using a dry plasmaenhanced chemical vapour deposition (PECVD) technique on a p-doped silicon wafer. The bottom gate contacts were patterned using e-beam lithography (EBL) and a $2 \mathrm{~nm} / 80 \mathrm{~nm} \mathrm{Cr} / \mathrm{Pd}$ stack was deposited using e-beam evaporation. The $30-\mathrm{nm} \mathrm{HfO}_{2}$ blocking oxide was grown by thermal atomic layer deposition (ALD) using TEMAH (tetramethylammonium hydroxide) and water as precursors. The floating gate was patterned similarly by EBL and a 5-nm-thick Pt layer was deposited using e-beam evaporation. Using the same process as described earlier, a 7-nm-thick $\mathrm{HfO}_{2}$ tunnel barrier was grown. The $\mathrm{MoS}_{2}$ is transferred on top of the tunnel barrier. To define the active region, PMMA polymer was used and patterned by EBL. The exposed area was then etched by oxygen plasma. Finally, drain-source contacts were patterned by EBL and a $2 \mathrm{~nm} / 100 \mathrm{~nm}$ thick Ti/Au stack was deposited using e-beam evaporation. Each die has 8 devices with a density of 0.386 devices per $10 \mu \mathrm{m}^{2}$. Resulting FGFETs have a typical channel length of $1 \mu \mathrm{m}$ and a width of $7.5 \mu \mathrm{m}$. A cross-sectional TEM image of a representative device is shown in Extended Data Fig. 12.

\section{Logic-in-memory fabrication}

A 270-nm-thick $\mathrm{SiO}_{2}$ layer is thermally grown using a dry PECVD technique on a p-doped silicon wafer. The bottom gate contacts were patterned using an MLA150 Advanced Maskless Aligner and a $2 \mathrm{~nm} / 40 \mathrm{~nm}$ $\mathrm{Cr} / \mathrm{Pt}$ stack was deposited using e-beam evaporation. The $30-\mathrm{nm}$ $\mathrm{HfO}_{2}$ blocking oxide was grown by thermal ALD using TEMAH and water as precursors. The floating gate was patterned by EBL and a 5-nm-thick Pt layer was deposited using e-beam evaporation. Using the same process as described earlier, a 7-nm-thick $\mathrm{HfO}_{2}$ tunnel barrier was grown. Prepatterned pads were exposed using the Advanced Maskless Aligner and $2 \mathrm{~nm} / 60 \mathrm{~nm}$ Ti-Au were deposited using e-beam evaporation. The $\mathrm{MoS}_{2}$ continuous film was transferred on top of the tunnel barrier. To define the active region, PMMA polymer was used and patterned by EBL. The exposed area was then etched by oxygen plasma. Finally, drain-source contacts were patterned by EBL and a $2 \mathrm{~nm} / 100 \mathrm{~nm}$ thick Ti/Au stack was deposited using e-beam evaporation. Resulting FGFETs have a typical channel length of $1 \mu \mathrm{m}$ and a width of $12.5 \mu \mathrm{m}$.

\section{Memory characterization}

Memory characterization is performed in high vacuum after in situ annealing at $120^{\circ} \mathrm{C} . I-V$ curve acquisition and pulse programming are performed using an Agilent E5270B mainframe with E5287A-ATO and E5281B-FG modules. A $10 \mathrm{pF}$ load capacitor is used for simulating the input capacitance of a cascade of logical stages in both FGFET inverter and FGFET NOR time measurements.

\section{Logic-in-memory}

The logic measurements of a two-input and a three-input unit cell, and a three-input NOR, were performed in air in a custom-built programmer using NI ELVIS II Board I/O. A detailed description of the programmer is given in Supplementary Note 9.

\section{Data availability}

The data that support the findings of this study are available at http:// doi.org/10.5281/zenodo.4073060.

42. Dumcenco, D. et al. Large-area epitaxial monolayer $\mathrm{MoS}_{2}$. ACS Nano 9, 4611-4620 (2015).

Acknowledgements We thank Z. Benes (CMI) for help with electron-beam lithography, L. Navrátilová (CIME) for the preparation of the device cross-section and R. Zamani (CIME) for help with TEM imaging. We acknowledge support from the European Union's Horizon 2020 
research and innovation programme under grant agreements 829035 (QUEFORMAL), 785219 and 881603 (Graphene Flagship Core 2 and Core 3), from the Marie Curie-Sklodowska COFUND (665667), from the H2O2O European Research Council (ERC, grant 682332) as well as from the CCMX Materials Challenge grant 'Large area growth of $2 \mathrm{D}$ materials for device integration'. Device preparation was carried out in part in the EPFL Centre of

MicroNanotechnology (CMI). TEM imaging was carried out in the EPFL Interdisciplinary Centre for Electron Microscopy (CIME).

Author contributions A.K. initiated and supervised the work. G.M.M. performed the device fabrication with initial assistance of Y.Z. G.M.M. constructed the characterization setup and performed electrical measurements. Y.Z. prepared the MOCVD grown $\mathrm{MoS}_{2}$ monolayers. Z.W. performed Raman spectroscopy and growth of wafer-scale films, supervised by A.R. M.T. performed HRTEM measurements and simulations. G.M.M., A.A. and A.K. analysed the data and wrote the manuscript with input from all authors.

Competing interests The authors declare no competing interests.

Additional information

Supplementary information is available for this paper at https://doi.org/10.1038/s41586-0202861-0.

Correspondence and requests for materials should be addressed to A.K.

Peer review information Nature thanks Devin Verreck, Takhee Lee and the other, anonymous, reviewer(s) for their contribution to the peer review of this work.

Reprints and permissions information is available at http://www.nature.com/reprints. 


\section{Article}

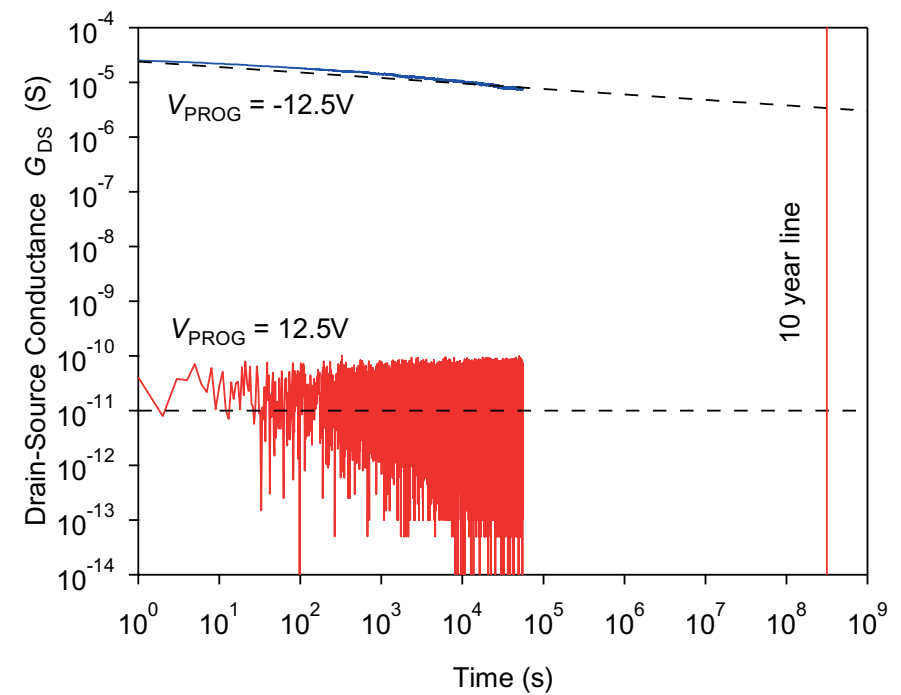

Extended Data Fig. 1 | Two-state retention time. The drain-source conductance $G_{\mathrm{DS}}$ is shown versus time. Blue curve, $V_{\mathrm{PROG}}=-12.5 \mathrm{~V}$; red curve, $V_{\text {PROG }}=+12.5 \mathrm{~V}$. To predict the trend of the decay, we fit both curves using the following expression, $f(x)=A x^{k}$ (dashed black lines). We expect that the device has a 10-year retention. 

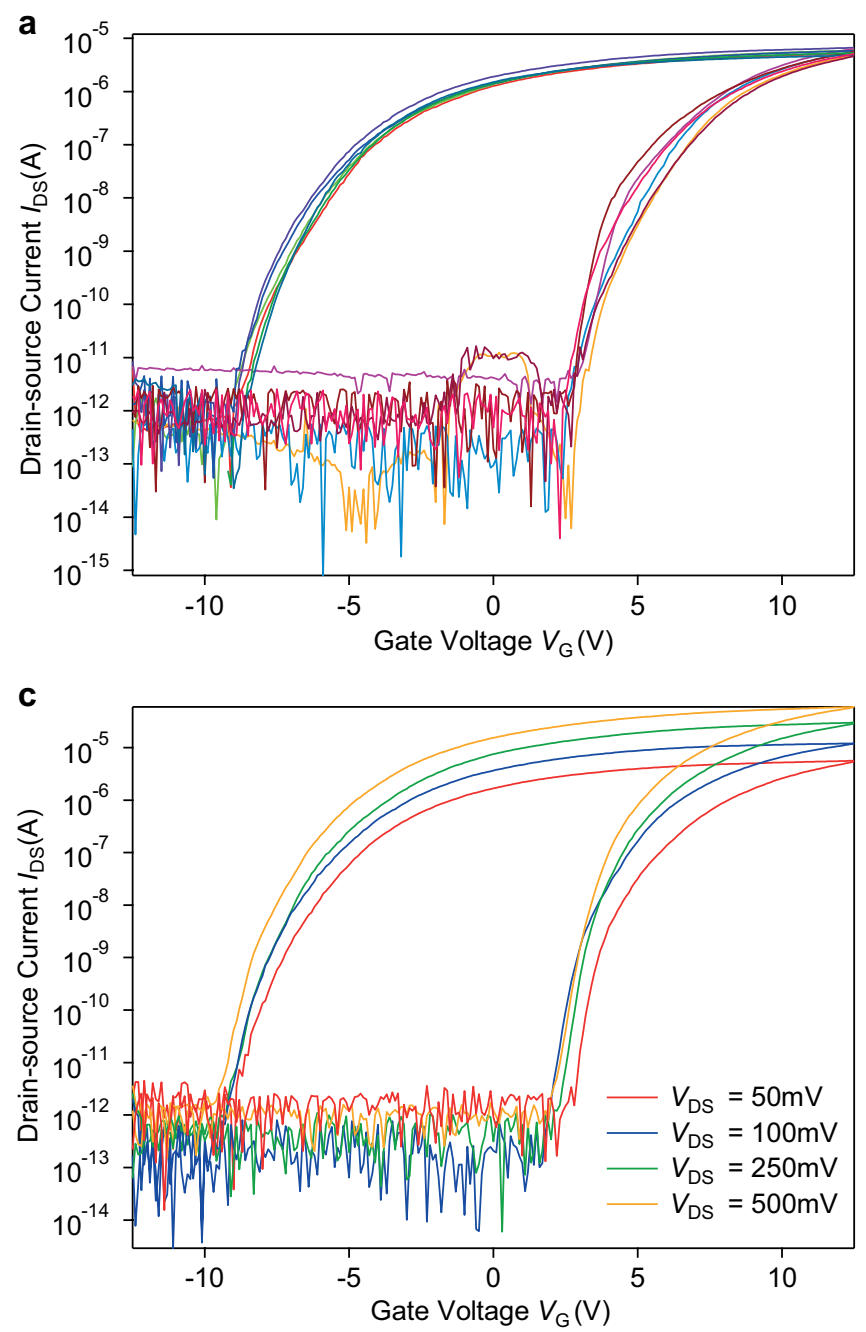

Extended Data Fig. 2 | Additional characteristics of MoS $_{2}$ FGFETs. a, Device variability. $I_{\mathrm{DS}}$ versus $V_{\mathrm{G}}$ curves for six different devices on the same die. b, Fresh $I_{\mathrm{DS}}$ versus $V_{\mathrm{G}}$ curves, corresponding to the first $V_{\mathrm{G}}$ sweep carried out on these devices. Maximal gate voltage $\pm V_{\mathrm{G}, \mathrm{MAX}}$ (corresponding to $V_{\mathrm{PROG}}$ ) is insufficient for inducing charge transfer into the floating gate memory. This shows the behaviour of the FGFET in the initial state. $c, I_{\mathrm{DS}}$ versus $V_{\mathrm{G}}$ for different values of $V_{\mathrm{DS}}$ (red curve, $50 \mathrm{mV}$; blue curve, $100 \mathrm{mV}$; green curve, $250 \mathrm{mV}$; orange curve,
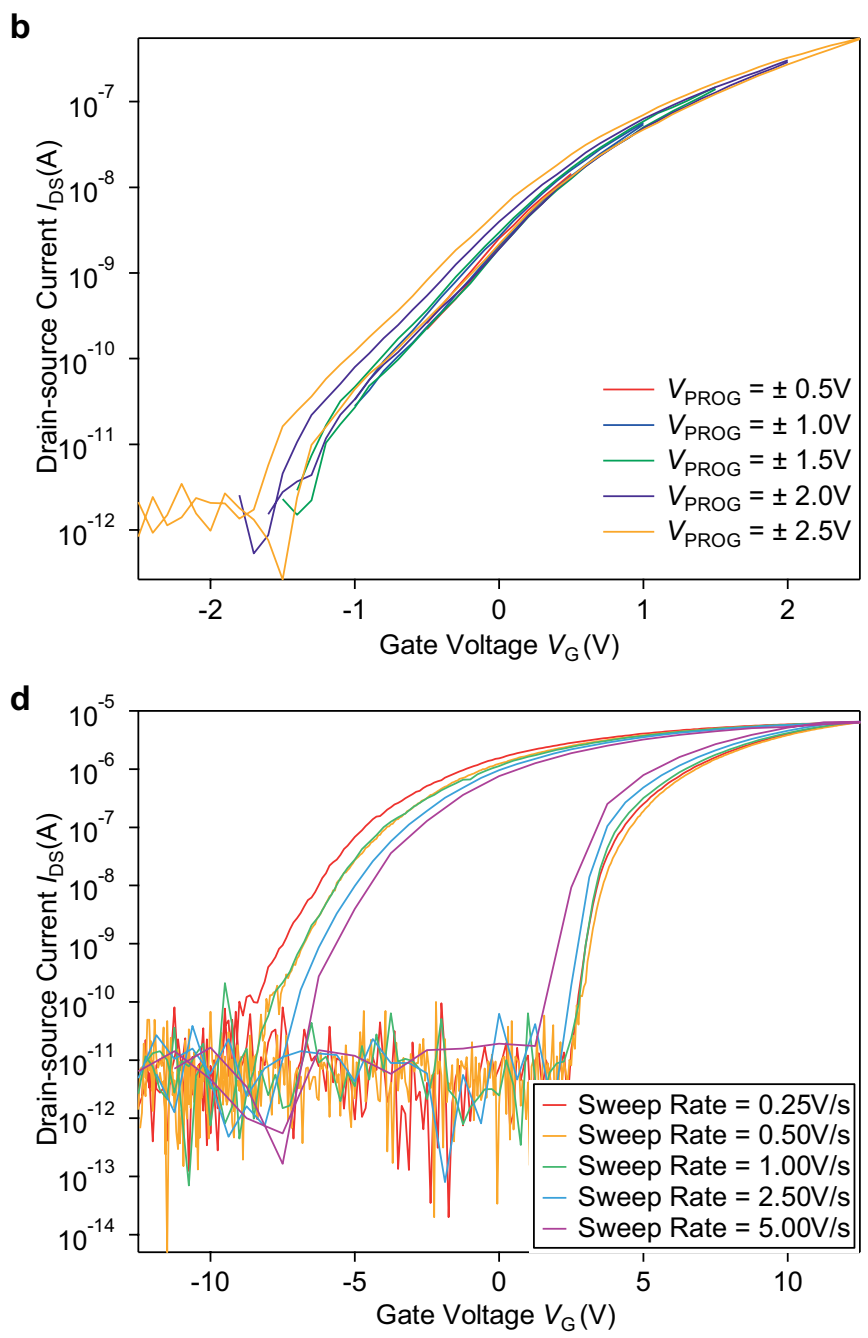

$500 \mathrm{mV}$ ). The progressive increase of the current without a decrease in the memory window demonstrates that the memory effect is not due to capacitive charges in the contacts. $d, I_{D S}$ versus $V_{\mathrm{G}}$ for different sweep rates. The decrease of the memory window is a function of the sweep rate. The decrease is most probably a result of charge dynamics limiting the charging and discharging of the floating gate. 
vacuum level

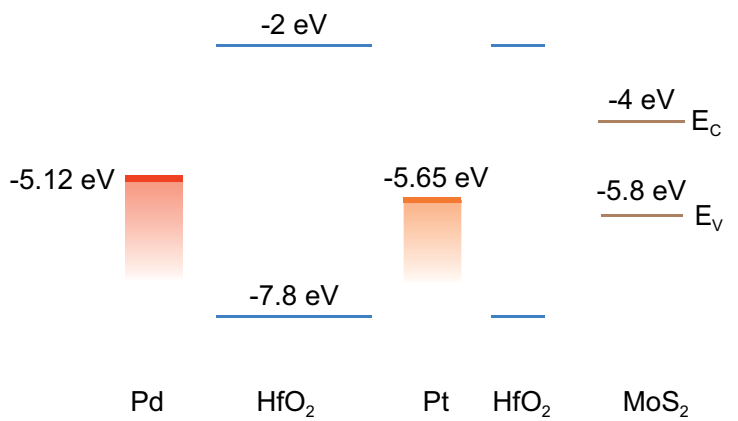

b

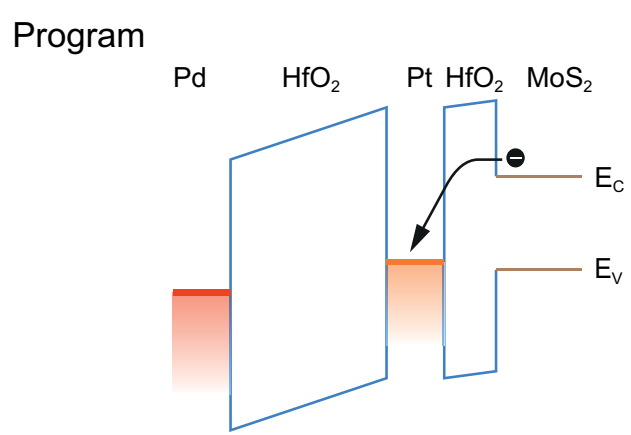

C

\section{Erase}

$\mathrm{Pd} \quad \mathrm{HfO}_{2} \quad \mathrm{Pt} \mathrm{HfO} \mathrm{HoS}_{2}$
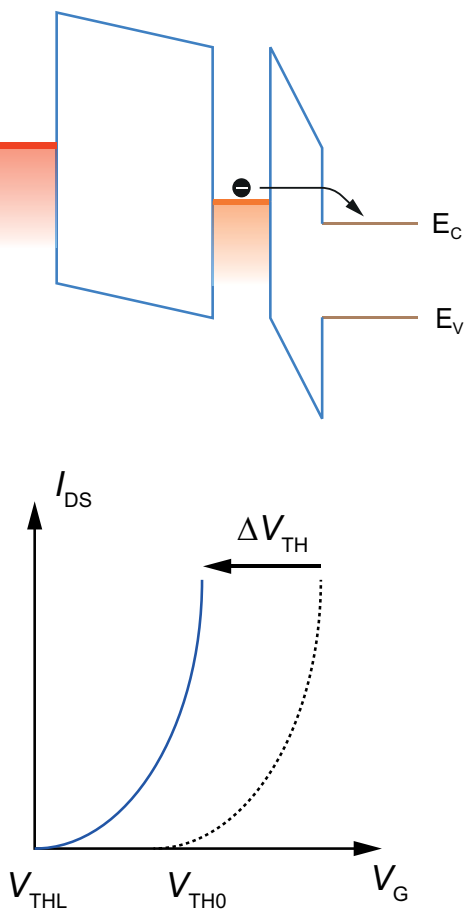

floating gate with the application of a positive gate voltage (upper panel). Lower panel, accompanying positive shift in the threshold voltage.c, Erase operation with electron extraction from the floating gate under the application of a negative gate voltage (upper panel). Lower panel, accompanying negative shift in the threshold voltage. conduction band and the top of the valence band, respectively.

b, Programming of the floating-gate memory by electron injection into the 
a

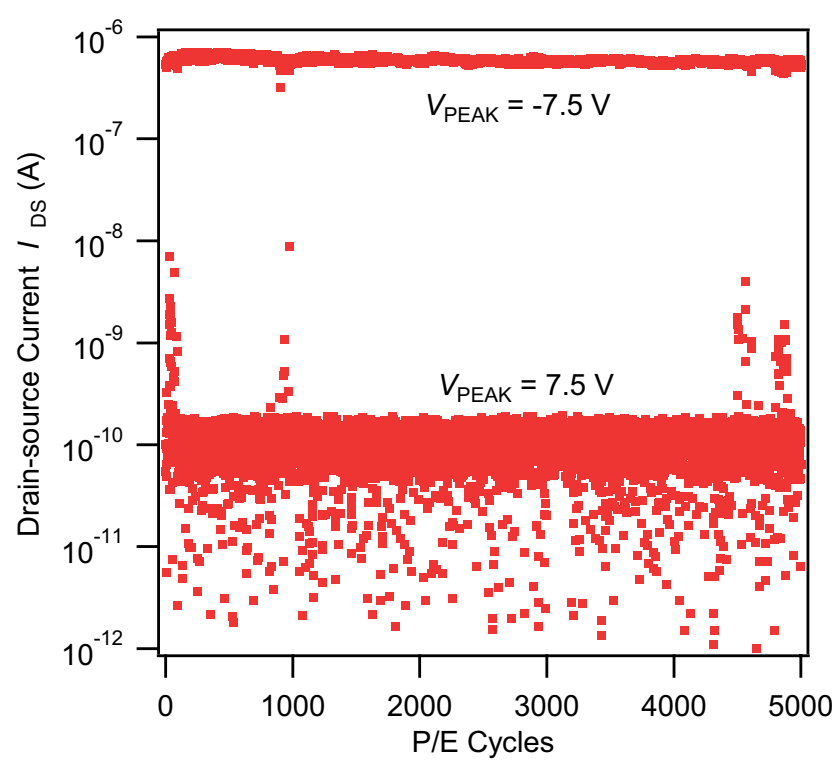

Extended Data Fig. 4 | Floating-gate endurance test. $I_{\mathrm{DS}}$ is shown as a

function of the number of program/erase (P/E) cycles. a, Each P/E cycle consists of a $100-\mathrm{ms}+7.5 \mathrm{~V}$ pulse for the erase operation, and a $100-\mathrm{ms}-7.5 \mathrm{~V}$ pulse for

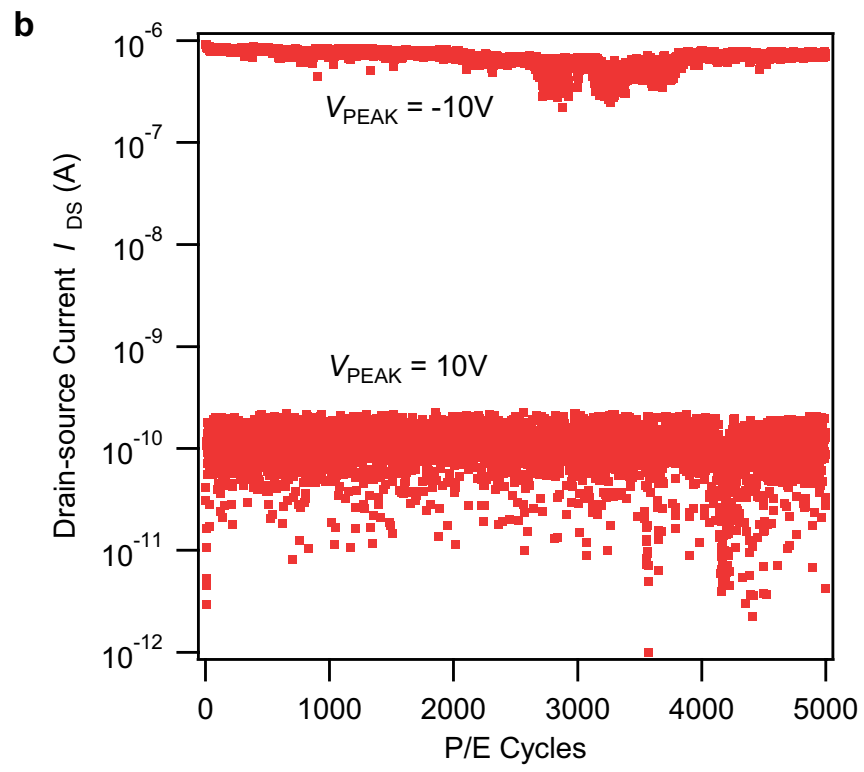

the program operation. $\mathbf{b}$, As a but with $\mathbf{a}+10.0 \mathrm{~V}$ pulse for erasing and $\mathbf{a}-10.0 \mathrm{~V}$ pulse for programming. Both measurements are taken using a constant $V_{\mathrm{DS}}=50 \mathrm{mV}$ and on the same device. 


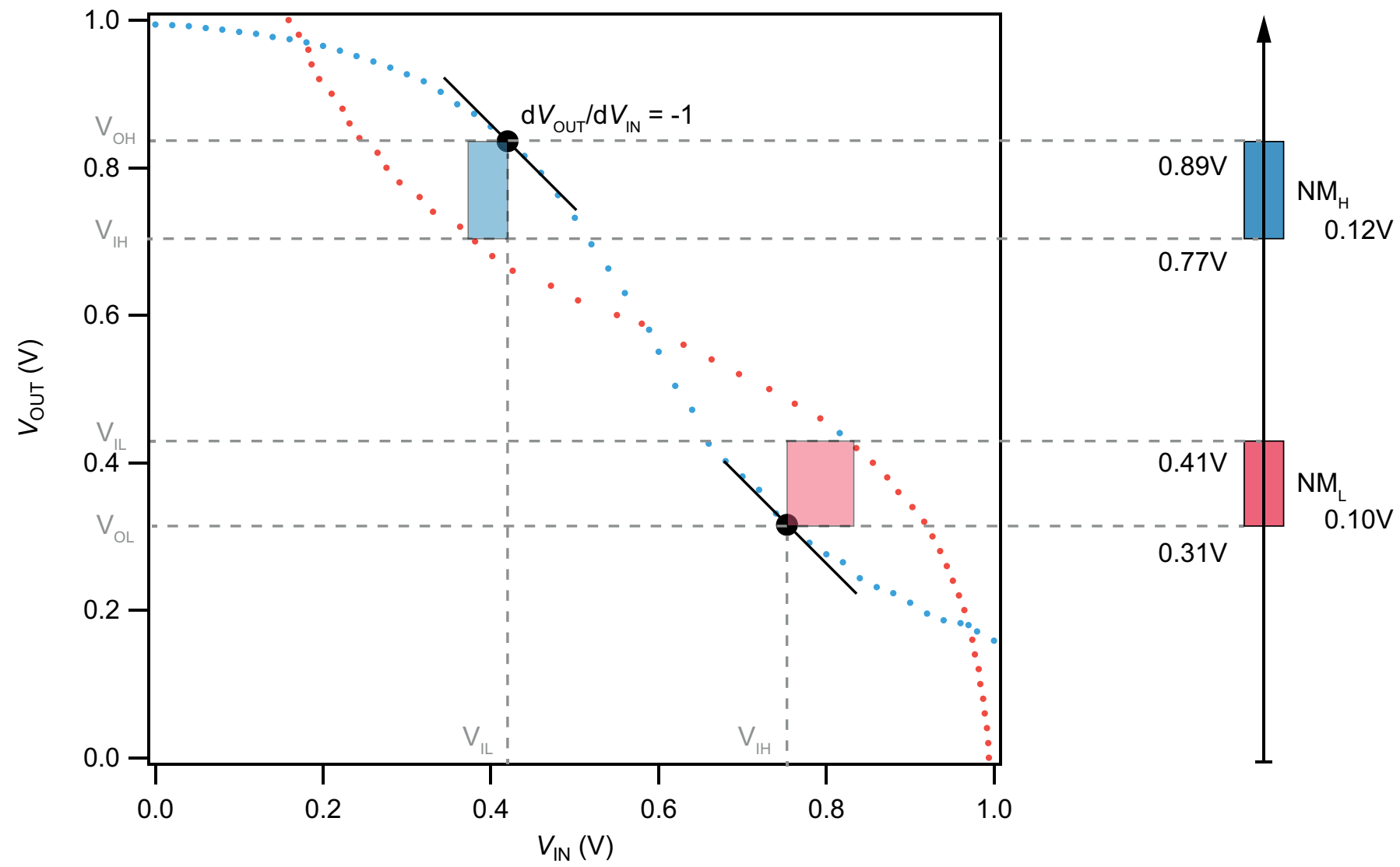

Extended Data Fig. 5 | Example of the graphical estimation of the noise margin for the inverter programmed with $\boldsymbol{V}_{\mathrm{PROG}}=8.5 \mathrm{~V}$. Output voltage $V_{\text {OUT }}$ as a function of input voltage $V_{\mathrm{IN}}$ (blue dots), and its mirror reflection (red dots).
$V_{\mathrm{OH}}$ and $V_{\mathrm{OL}}$ are defined as the points where the slope of the transfer curve ( $V_{\mathrm{OUT}}$ as a function of $V_{\mathrm{IN}}$, blue dots) is equal to -1 , whereas $V_{\mathrm{IL}}$ and $V_{\mathrm{IH}}$ are the corresponding values of $V_{\mathrm{IN}}$. 


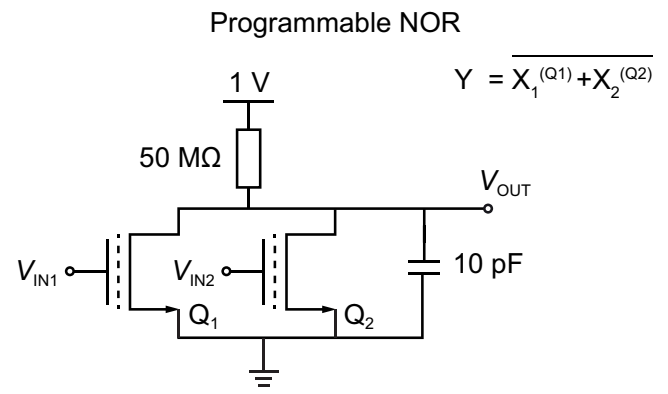

b

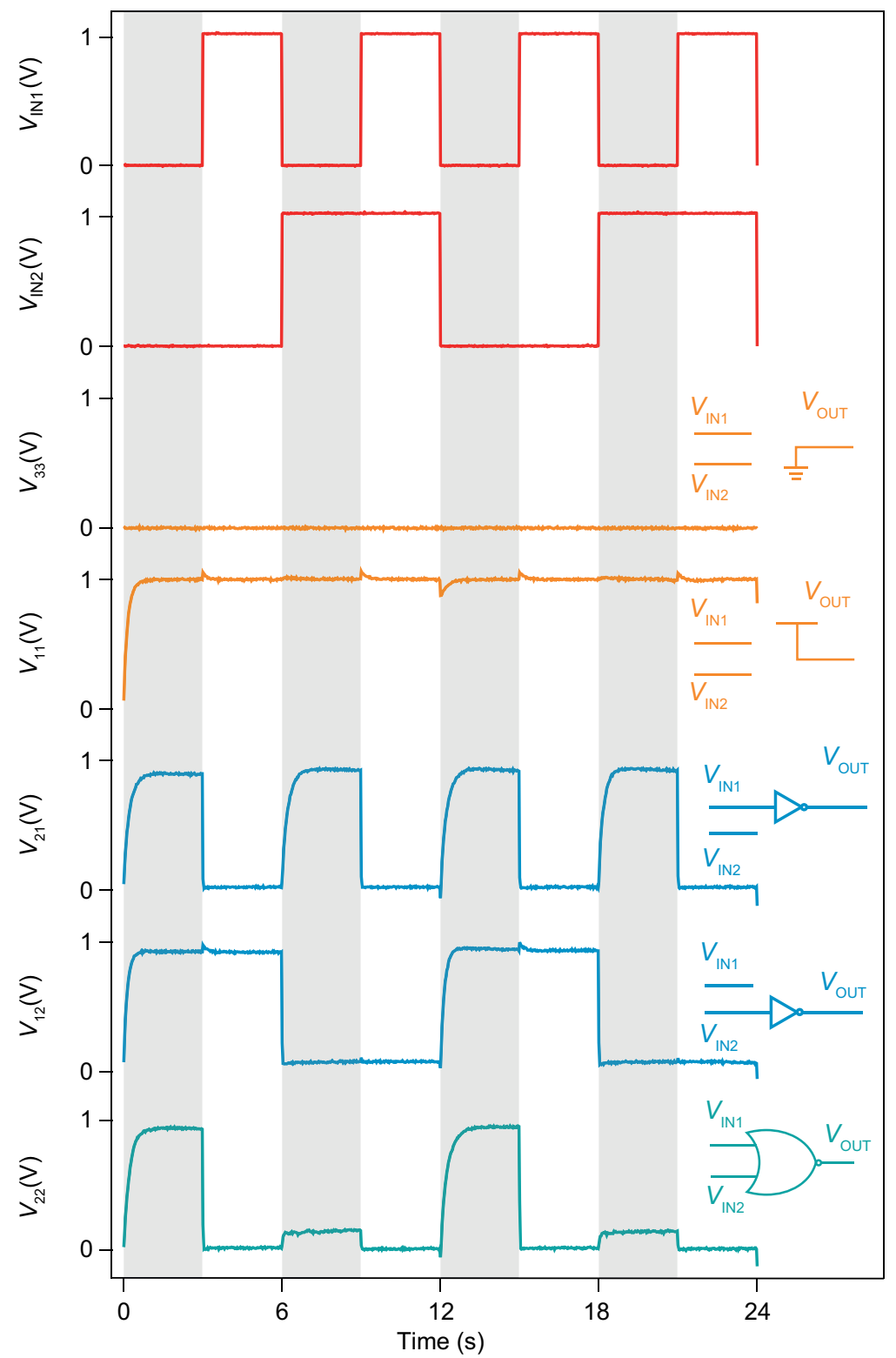

Extended Data Fig. 6 | Circuit schematic and logic for a two-input NOR. a, Circuit schematic for a two-input NOR. b, Logic over time for different Blue: output curves for $Q_{1,2}=21$, inverter A (IN1); $Q_{1,2}=12$, inverter B (IN2). Green: output curve for $Q_{1,2}=22$, NOR A,B. (Here and in Extended Data Figs. 7, 8, we programming states $Q_{1}, Q_{2}$. Red: time traces of input voltages $V_{\mathrm{IN1}}$ and $V_{\mathrm{IN} 2}$. denote (for example) programming state ' $Q_{1}=3, Q_{2}=3^{\prime}$ by ' $Q_{1,2}=33^{\prime}$.). 
Programmable 3-input NOR
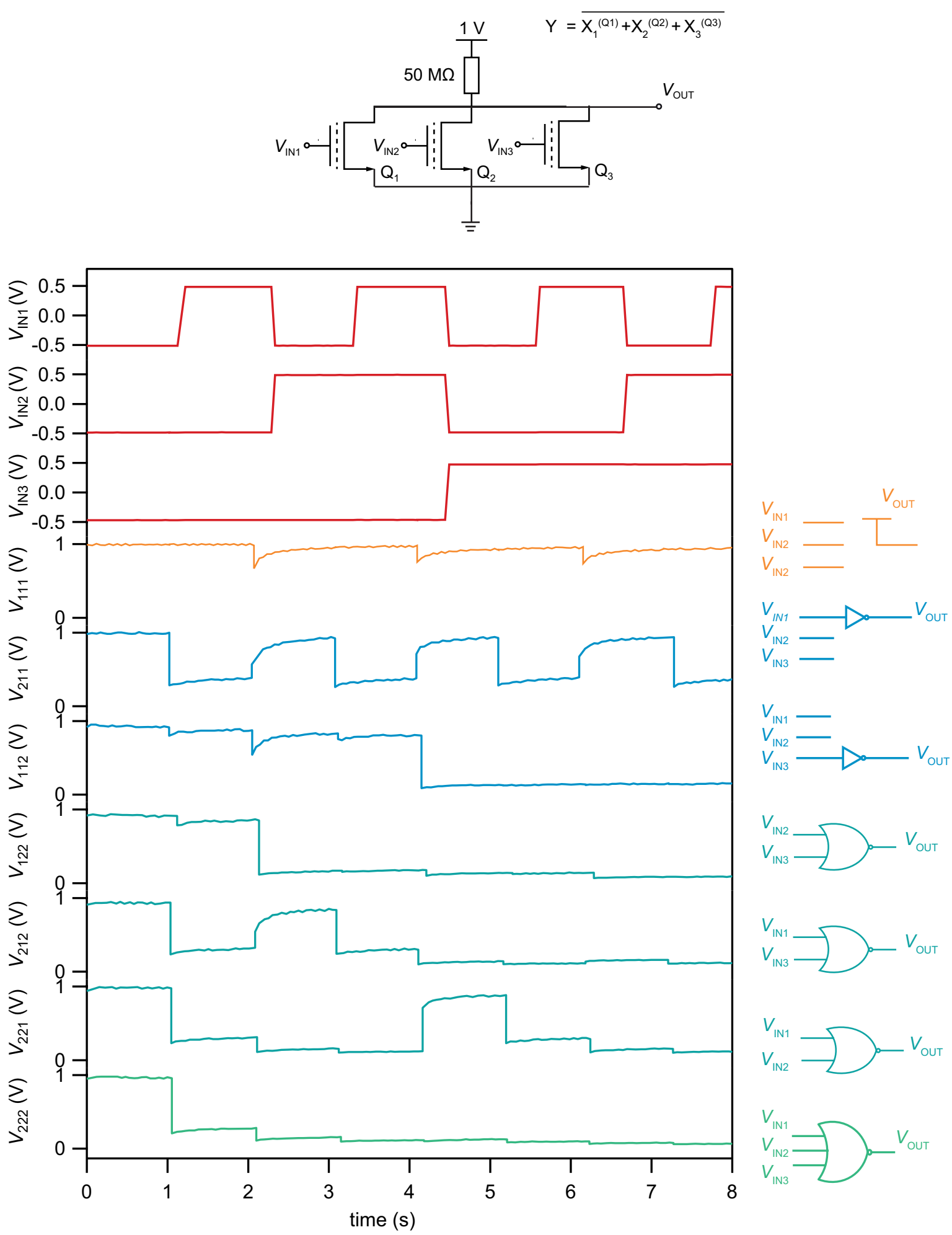

Extended Data Fig. $7 \mid$ Three-input NOR. a, Circuit schematic for a three-input NOR. b, Logic over time for different programming states $Q_{1}, Q_{2}, Q_{3} \cdot Q_{1-3}=111$, constant HIGH; $Q_{1-3}=211$, inverter A (IN1); $Q_{1-3}=112$, inverter C (IN2); $Q_{1-3}=122$, NOR B,C; $Q_{1-3}=212$, NOR A,C; $Q_{1-3}=221$, NOR A,B; $Q_{1-3}=222$, NOR A,B,C. 
a

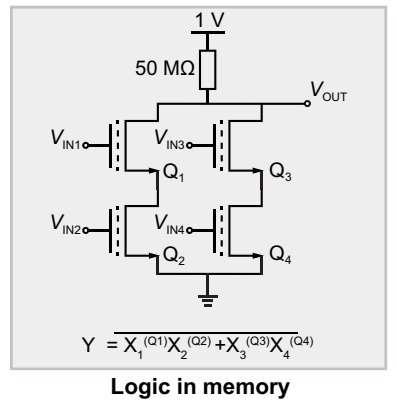

b

Interfaces

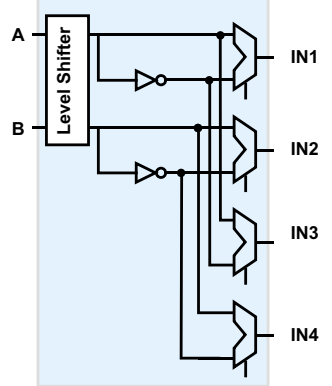

Extended Data Fig. 8 | Two-input logic-in-memory concept and interpretation.a, Two-input schematic of the logic-in-memory concept.

b, Interface model for input polarity control. c, NAND gate, $Q_{1-4}=2211$; d

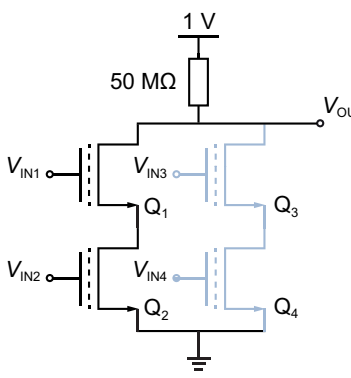

$Q_{1}, Q_{2}:$ State 2

$Q_{3}, Q_{4}$ : State 1

$I_{1}=I N_{3}=A ; I N_{2}=I N_{4}=B$;

$Y=\overline{A \cdot B}$

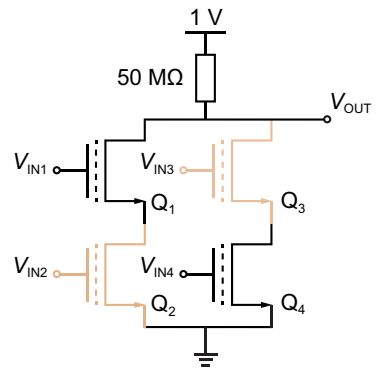

$Q_{1}, Q_{4}:$ State 2

$\mathbb{I N}_{1}=\mathbb{I N}_{3}=A ; I_{2}=I_{4}=B ;$

$Y=\overline{A+B}$ e

XOR

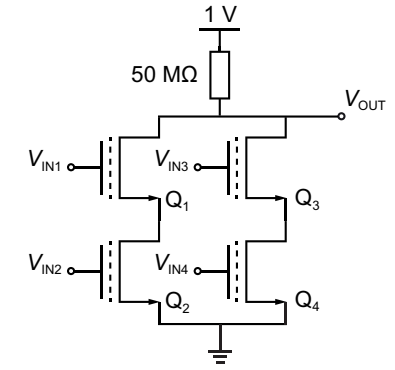

$\mathrm{Q}_{1}, \mathrm{Q}_{2}, \mathrm{Q}_{3}, \mathrm{Q}_{4}$ : State 2

$I N_{1}=A ; I N_{2}=B ; I N_{3}=\bar{A} ; I N_{4}=\bar{B} ;$

$Y=\overline{A \cdot B+\bar{A} \cdot \bar{B}}$

$Y=\overline{A \cdot B+\overline{(A+B)}}$

$Y=\overline{(A \cdot B)} \cdot \overline{\overline{(A+B)}}$

$Y=(\bar{A}+\bar{B}) \cdot(A+B)=\bar{A} \cdot B+A \cdot \bar{B}$ d, NOR gate, $Q_{1-4}=2332 ; \mathbf{e}, X O R$ gate, $Q_{1-4}=2222$. We derive the XOR canonical form by applying De Morgan's laws. 
a

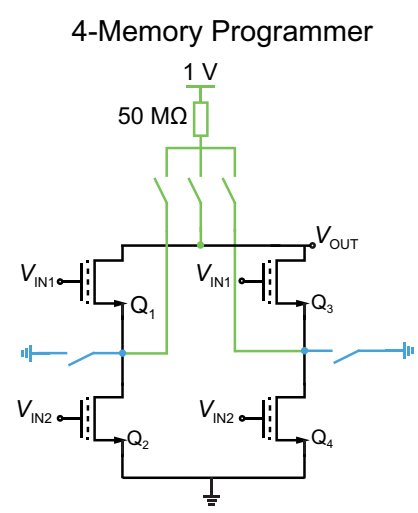

Logic Equation:

$$
Y=\overline{X_{1}^{(Q 1)} X_{2}^{(Q 2)}+X_{3}^{(Q 3)} X_{4}^{(Q 4)}}
$$

b

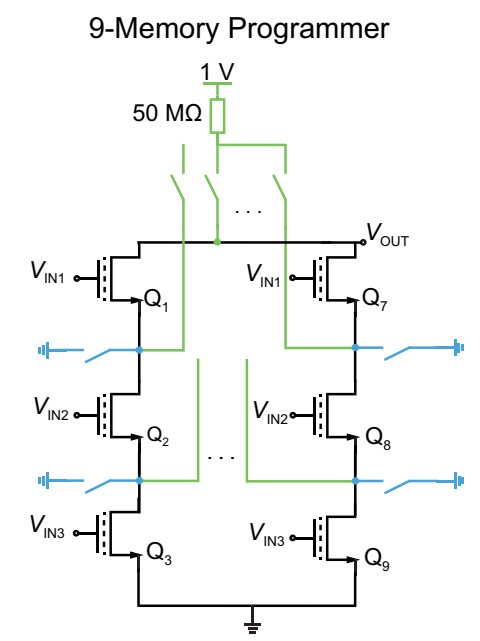

Logic Equation:

$$
Y=\frac{\overline{X_{1}^{(Q 1)} X_{2}^{(Q 2)} X_{3}^{(Q 3)+}}}{X_{4}^{\left({ }^{(Q 4)} X_{5}{ }^{(Q 5)} X_{6}{ }^{(Q 6))}+\right.}}
$$

C

Programmer Block Diagram

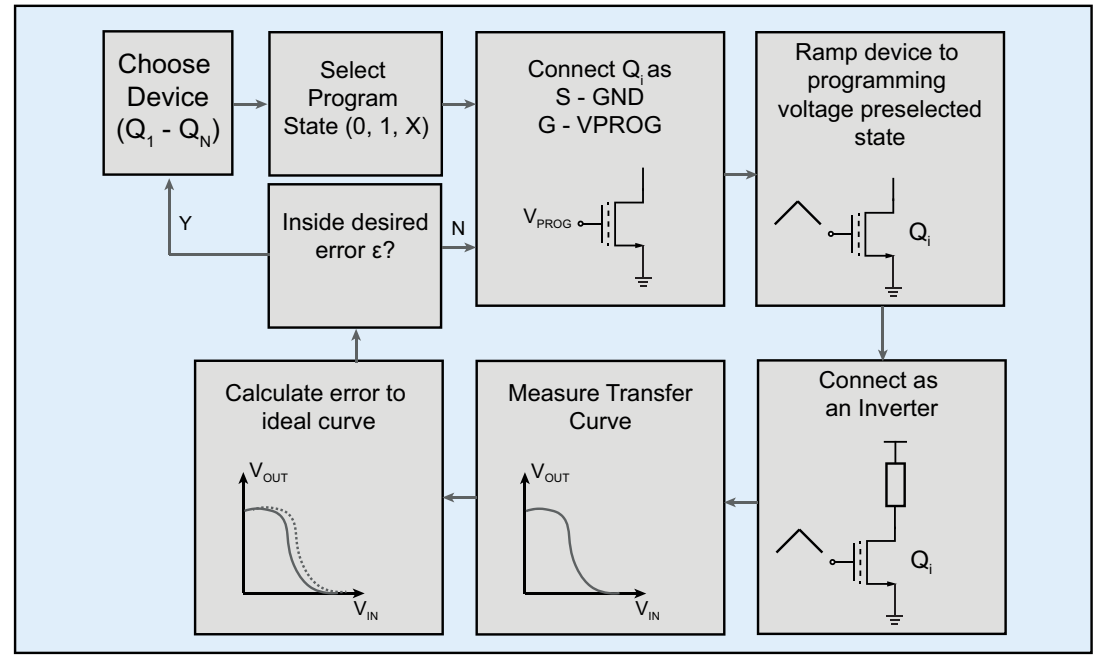

Test Block Diagram

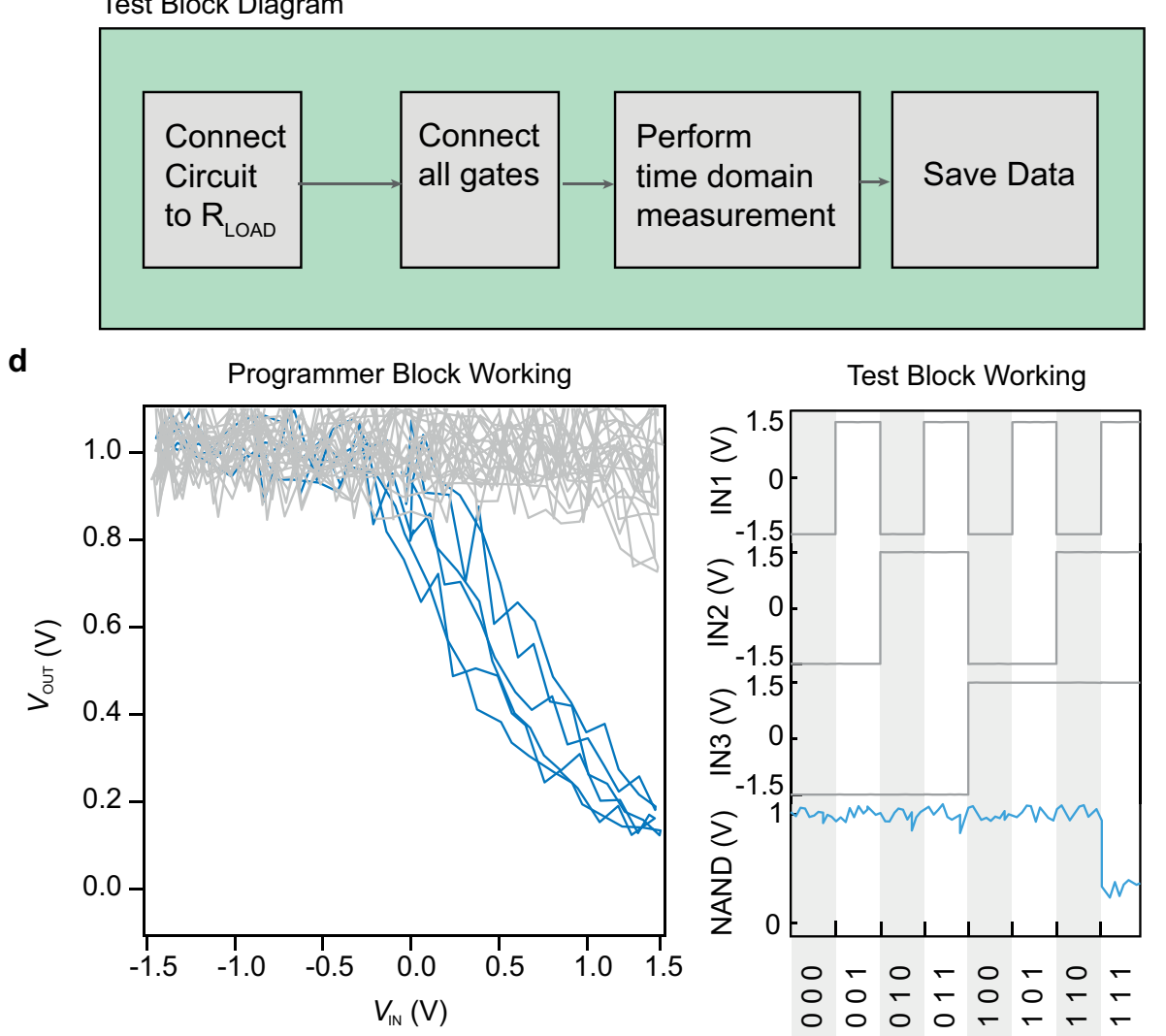

Extended Data Fig. 9 | Hardware and software implementation of the logic-in-memory programmer. a, b, Hardware implementation of the four-memory programmer (a) and of the nine-memory programmer $(\mathbf{b})$. c, Software working diagram of the programming (top) and test (bottom) blocks. d, Example of programming (left) and test (right) blocks working, using a nine-memory programmed into the following state $Q_{1-9}=222111111$ to perform a three-input NAND operation. 


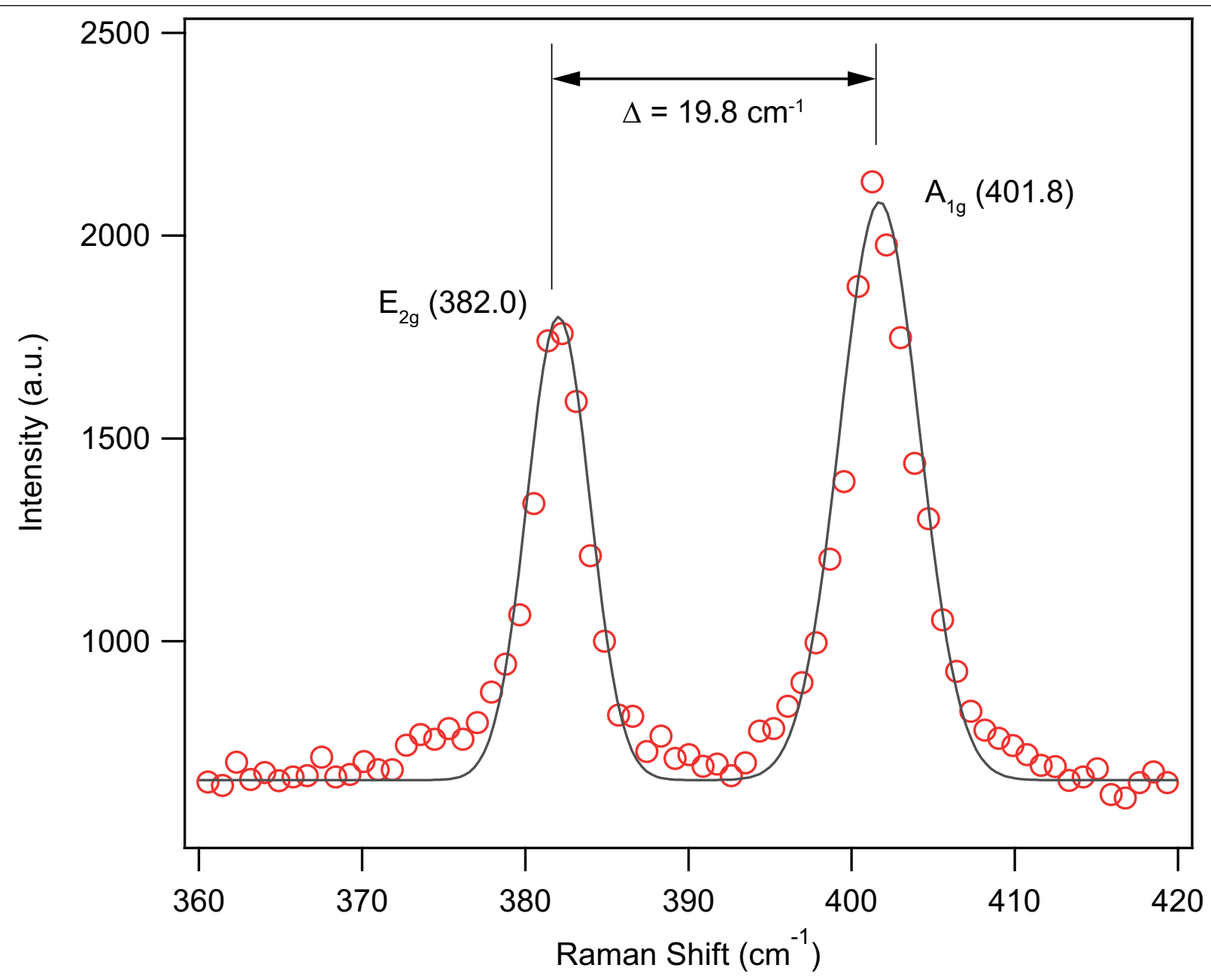

Extended Data Fig. 10 | Raman characterization of monolayer MoS $_{\mathbf{2}}$. Raman spectrum of transferred $\mathrm{MoS}_{2}$ from a single crystal (which also provided the grating. The observed wavenumber difference between the $A_{1 g}$ and $E_{2 g}$ Raman modes of $\mathrm{MoS}_{2}$ is consistent with a monolayer. Black line is a fit to the data points (red circles). 


\section{Article}
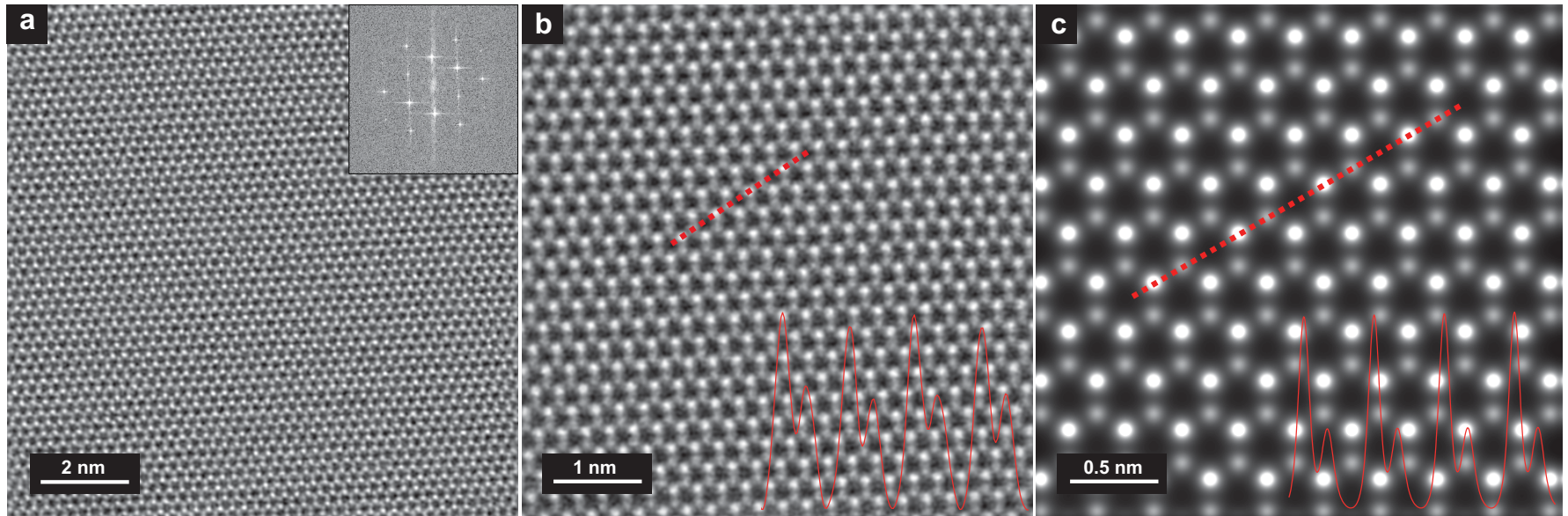

Extended Data Fig. $11 \mid$ ADF-STEM images of monolayer $\operatorname{MoS}_{\mathbf{2}}$. a, Atomically resolved STEM image showing a large region of monolayer $\mathrm{MoS}_{2}$. Inset, fast Fourier transform (FFT) amplitude spectrum further shows the crystalline monolayer $\mathrm{MoS}_{2}$ structure. b, A magnified filtered STEM image taken from a shows the $2 \mathrm{H}$ crystal structure of monolayer $\mathrm{MoS}_{2}$ c, STEM simulation image of monolayer $\mathrm{MoS}_{2}$. The intensity line profiles at bottom right of $\mathbf{b}$ and $\mathbf{c}$ are taken along the dashed lines in those images, and show the peak positions of Mo atoms and $\mathrm{S}$ atoms. 

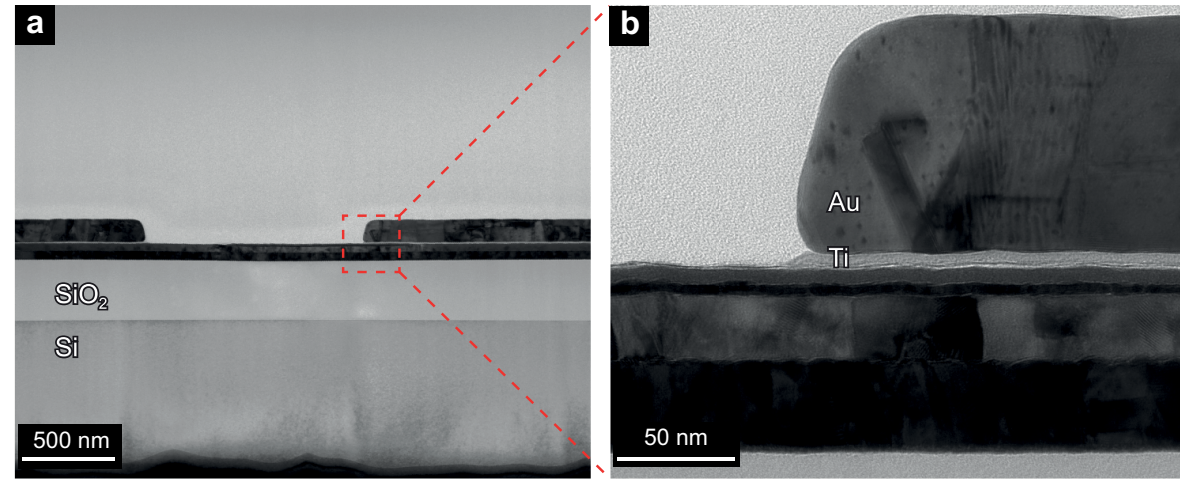

Extended Data Fig. 12 | FGFET TEM cross-section. a, Wide-field view of the device fabricated using the logic-in-memory process. $\mathbf{b}$, Magnified view of the contact area boxed in a. c, Cross-section image of the gate stack consisting of

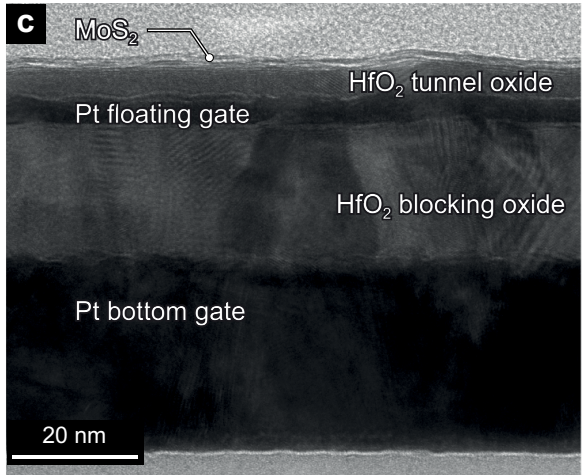

(from bottom to top) Pt bottom gate, $\mathrm{HfO}_{2}$ blocking oxide, Pt floating gate, $\mathrm{HfO}_{2}$ tunnel oxide. The $\mathrm{MoS}_{2} 2 \mathrm{D}$ channel is on top of the gate stack. 